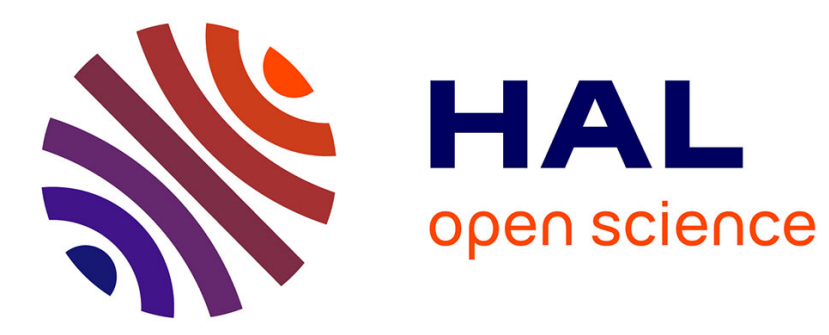

\title{
Direct numerical simulations of high velocity ratio coaxial jets: mixing properties and influence of upstream conditions
}

Guillaume Balarac, Mohamed Si-Ameur, Marcel Lesieur, Olivier Métais

\section{- To cite this version:}

Guillaume Balarac, Mohamed Si-Ameur, Marcel Lesieur, Olivier Métais. Direct numerical simulations of high velocity ratio coaxial jets: mixing properties and influence of upstream conditions. Journal of Turbulence, 2007, 8, pp.N22. 10.1080/14685240600833094 . hal-00261424

\section{HAL Id: hal-00261424 \\ https://hal.science/hal-00261424}

Submitted on 19 Mar 2020

HAL is a multi-disciplinary open access archive for the deposit and dissemination of scientific research documents, whether they are published or not. The documents may come from teaching and research institutions in France or abroad, or from public or private research centers.
L'archive ouverte pluridisciplinaire HAL, est destinée au dépôt et à la diffusion de documents scientifiques de niveau recherche, publiés ou non, émanant des établissements d'enseignement et de recherche français ou étrangers, des laboratoires publics ou privés.

\section{(c)(1)}

Distributed under a Creative Commons Attribution| 4.0 International License 


\title{
Direct numerical simulations of high velocity ratio coaxial jets: mixing properties and influence of upstream conditions
}

\author{
G. BALARAC, ${ }^{*}$ M. SI-AMEUR, M. LESIEUR and O. MÉTAIS \\ Equipe MoST/LEGI. B.P. 53, 38041 Grenoble Cedex 09, France
}

\begin{abstract}
Direct numerical simulations (DNS) are performed to investigate mixing in free round coaxial jets. A great attention has been put on the influence of upstream conditions upon the global flow structure and the mixing process. The mixing behavior is studied through the spatial and temporal development of the mixture fraction of the annular and the inner fluids, and examined by means of flow visualization and statistics. It is shown that the turbulent mixing process and the mixture fraction field in coaxial jets depend on the upstream conditions, even though a quasi self-similar state is reached. The mixing alterations are explained by the understanding of the flow dynamics modifications implied by the different upstream conditions. These alterations are mainly due to the intense generation of streamwise vortices, favored by high inlet velocity gradients and velocity ratios, as well as low ratios between the inner and the outer jet diameters. This is associated with a high quality of mixing, as far as global mixedness is concerned. It is also shown that the annular fluid reaches the inner fluid and mixes swiftly into it. Conversely, the latter remains confined. Additionally, spots of pure unmixed species are observed at the end of the computational domain, and shown to be due to the annular jet.
\end{abstract}

Keywords: Turbulence; Coaxial jets; Mixing properties

\section{Introduction}

Coaxial jets are widely used in industrial applications, especially in chemical engineering systems and combustion devices. They are for instance linked to flames in power producing gas turbines. Achievement of high rates of molecular mixing between turbulent gaseous jets is a challenging target. A better mixing is of crucial importance to improve combustion efficiency and reduce pollutant emissions in such technological combustion systems.

In this framework, the present work aims to study qualitatively and quantitatively scalar mixing in free round turbulent coaxial jets, with emphasis put on modifications of the upstream conditions. The key interest is to understand these influences as a preliminary attempt to predict the mixing behavior in practical coaxial nozzles for which typical initial conditions are not known. In these situations, mixing is subject to many interacting processes and takes place at all turbulent scales. We will speak here of one kind of mixing, the macro-mixing which is controlled by the dynamics of turbulent eddies. A detailed topological analysis of such structures has already been done in earlier works [1,2]. Micro-mixing is relevant when the molecular mixing is deemed and is evaluated in terms of probability density functions (PDF).

In order to place the present study in its context, we present a literature survey. In fact few works have considered the important problem of mixing. The numerical investigation

\footnotetext{
*Corresponding author. E-mail: guillaume.balarac@hmg.inpg.fr
} 
of Salvetti [3] has examined the mixing process but the configuration was limited to an axisymmetric approximation. They have provided informations about the mixing evolution in the early stage of flow development. Jahnke et al. [4] have performed a large-eddy simulation (LES) of mixing in a confined coaxial jet. They study the influence of different parameters (such as Reynolds number, Schmidt number and density ratio), and consider the case where the inner jet is faster than the outer jet. In a previous experimental work, Villermaux and Rehab [5] have determined the mixing time scale as a function of the Schmidt number. Ferrao et al. [6] have also studied the turbulent scalar mixing in the initial region of an axisymmetric coaxial jet of $\mathrm{CO}_{2}$ and air. They have shown in particular that the structure of the inner jet is affected by fluid from both the inner and annular jet. Additional experiments [7-9] studied forced coaxial jets for an active mixing control. They provide a thorough instantaneous snapshot of the concentration field and statistical information on the concentration fluctuations. Ritchie et al. [7] have highlighted that the active mixing control by synthetic jets was able to influence the inner and outer jets.

In this paper, we present a study of near- and far-field structures of constant density round coaxial jets, with emphasis put on mixing properties. We restrict ourselves to mixing without chemical reactions, in order to concentrate on the effect of turbulent vortices and structures on the mixing process, with a particular focus on the influence of upstream conditions. A great attention is paid to the spatio-temporal evolution of the mixture fraction $f$, with a particular interest in PDF of $f$. The tool of investigation is DNS, which allows us to resolve the smallest scales and hence describes the molecular mixing process itself. Even though the present DNS is restricted to low Reynolds numbers (in order to resolve all the mixing scales), it can be considered as a noteworthy step for our developing work using LES at a high Reynolds number [10]. Furthermore, a nearly fully developed flow has been reached in the far field, as demonstrated by the flow dynamics and energy spectra. The maximal local Reynolds number approaches the minimal value required to produce a quasi fully developed turbulence [11], which gives confidence in tracking the mixing process through the increase of the interfacial area between the species which is due to the stirring process linked to the turbulent structures. A successful description and quantification of the mixing behavior in terms of marching and non-marching PDF provides valuable informations for modeling procedures, which are difficult to verify experimentally considering the constraints in measurements resolution. The outline of the paper is as follows. Section 2 presents the numerical method and computational parameters. Section 3 gives a picture of global mixing for the configuration selected as a reference case. Section 4 constitutes the main part of this paper showing the influence of the upstream conditions upon mixing.

\section{Numerical method and computational parameters}

We solve numerically in a parallelepipedic computational domain the incompressible NavierStokes equations written in Cartesian coordinates. The spatial discretization is performed with the aid of a sixth-order compact finite-difference scheme in the streamwise direction, together with pseudo-spectral methods in transverse and spanwise directions which are taken as periodic. Pressure-velocity coupling is assured by a fractional step method, requiring the solution of a Poisson equation to insure incompressibility of the velocity field. The advancement in time is carried out by a third-order Runge-Kutta scheme. The outlet boundary condition is of a non-reflective type where the convective and viscous terms are explicitly advanced [12]. Gonze [13] showed that the coherent structures are not distorted in the computational domain by this condition. Full numerical discretization details can be found in [14]. Moreover, we 


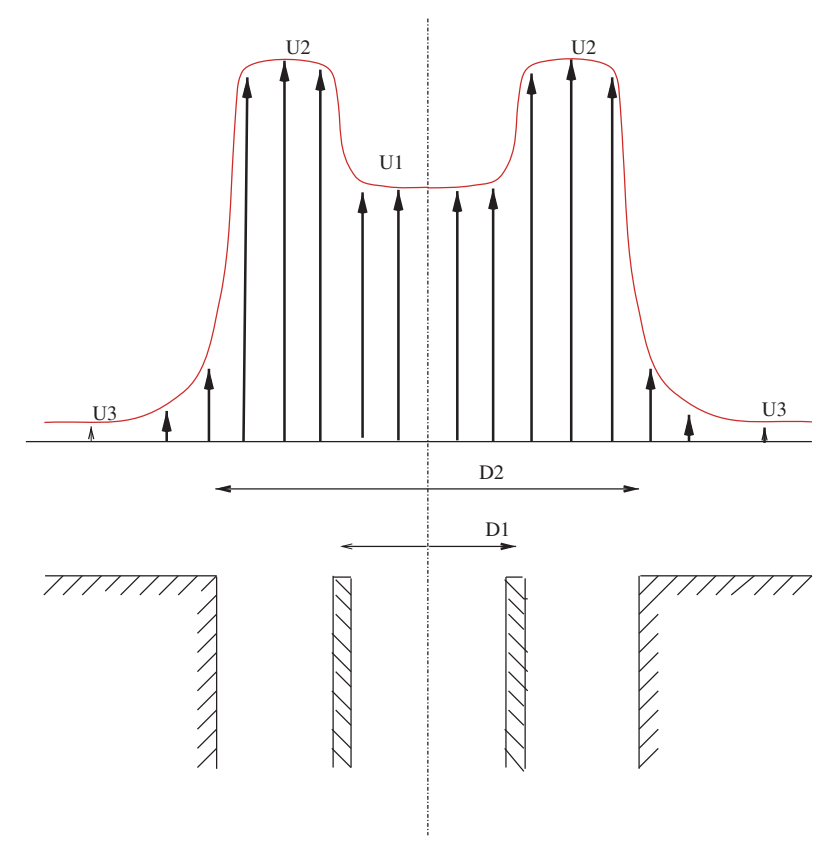

Figure 1. Sketch of the inlet velocity profile. $U_{1}$ is the inner jet velocity coming out of the nozzle (diameter $D_{1}$ ). $U_{2}$ is the velocity of the outer jet stream, issuing from a diameter $D_{2}>D_{1}$.

consider the mixture fraction $f$ to study the mixing between two species $\mathrm{A}$ and $\mathrm{B}$ seeded in the coaxial jets. Thus, $f=1$ (respectively 0 ) if only species $\mathrm{B}$ (respectively $\mathrm{A}$ ) is there. The evolution of the mixture fraction is given by a transport equation (convection-diffusion) which is solved simultaneously with the flow equations. For the spatial discretization of the convection term, we use a second-order semi-discretized TVD Roe scheme [15].

The shape of the inlet velocity profile is constructed with two hyperbolic-tangent profiles (see figure 1). More precisely, the inlet velocity profile $\vec{U}_{\text {med }}(r)=\left(U_{\text {med }}(r), V_{\text {med }}(r), W_{\text {med }}(r)\right)$ in Cartesian coordinates writes

$$
U_{\mathrm{med}}(r)=\left\{\begin{array}{ll}
\frac{U_{1}+U_{2}}{2}+\frac{U_{1}-U_{2}}{2} \tanh \left(\frac{r-R_{1}}{2 \theta_{01}}\right) & \text { for } r<R_{m} \\
\frac{U_{2}+U_{3}}{2}+\frac{U_{2}-U_{3}}{2} \tanh \left(\frac{r-R_{2}}{2 \theta_{02}}\right) & \text { for } r>R_{m}
\end{array},\right.
$$

with $V_{\text {med }}(r)=W_{\text {med }}(r)=0$. In equation (1), $U_{1}$ is the inner coaxial jet velocity, $U_{2}$ is the outer velocity and $U_{3}$ is a very small co-flow. Moreover, $R_{1}, R_{2}$ and $R_{m}=\left(R_{1}+R_{2}\right) / 2$ are the inner, outer and averaged radii, and $\theta_{01}$ and $\theta_{02}$ are the inlet momentum thicknesses of the inner and outer shear layers, respectively. At the beginning of the coaxial jet, a weak-amplitude random noise is superposed on the inlet velocity profile (roughly $3 \%$ of the fast outer stream). It is applied to the three velocity components (see [1]). The computational code has been validated in round jets [14], plane jets [16] and coaxial jets [1,2].

All simulations have been carried out on the same computational grid consisting in $231 \times$ $384 \times 384$ points with a uniform mesh size in all three directions for a domain size of $10.8 D_{1} \times$ $10.65 D_{1} \times 10.65 D_{1}$, along the streamwise $(x)$ and the two transverse directions $(y, z)$. In all the simulations the Reynolds number and the Schmidt number are $\operatorname{Re}=U_{2} R_{2} / v=3000$ and $S c=1$. To study the influence of the upstream conditions, we vary the velocity ratio, $r_{u}=U_{2} / U_{1}$, the initial momentum thicknesses $\theta_{01}$ and $\theta_{02}$, and the diameter ratio $\beta=D_{2} / D_{1}$. Table 1 summarizes the different flow configurations studied. The initial momentum-fluxes ratio between the outer and the inner jets is also given. The inlet mixture fraction profile is 
also built with hyperbolic tangents

$$
f(x=0, r, t)= \begin{cases}\frac{f_{1}+f_{2}}{2}+\frac{f_{1}-f_{2}}{2} \tanh \left(\frac{r-R_{1}}{2 \theta_{01}}\right) & \text { for } r<R_{m} \\ \frac{f_{2}}{2}+\frac{f_{2}}{2} \tanh \left(\frac{r-R_{2}}{2 \theta_{02}}\right) & \text { for } r>R_{m} .\end{cases}
$$

Note that the co-flow is always composed of species $\mathrm{A}(f=0)$, and we seed species $\mathrm{B}(f=1)$ either into the inner jet (in this case, $f_{1}=1$ and $f_{2}=0$ in equation (2)) or the outer jet (in this case, $f_{1}=0$ and $f_{2}=1$ in equation (2)). Of course, we seed species A in the jet where species B is not seeded. The different numerical simulations are called DNSXY, where $X$ is a number which stands for the kinematic parameters of the simulation and $Y$ is ' $\mathrm{O}$ ' or ' $\mathrm{I}$ ' if species B is seeded in the outer jet or the inner jet, respectively.

\section{Picture of global mixing}

In this section we review the main mechanisms of transition to turbulence and the mixing behavior in coaxial jets (see also [17]), through the DNS1O case (where species B is seeded in the annular jet). This configuration is selected as a reference case to study the influence of upstream conditions (velocity ratio, velocity-gradients profile, diameter ratio).

\subsection{Flow dynamics}

The main features of the flow can be obtained with an observation of the flow structures. Figure 2 shows positive $Q$ isosurfaces for DNS1O colored by the value of streamwise vorticity $\omega_{x}$ (see color scale in figure 2). Note that the green corresponds to a value near zero for $\omega_{x} \cdot Q$ is the second invariant of the velocity-gradient tensor. The positive $Q$ criterion was proposed by Hunt et al. [18] and is well recognized as a good coherent-vortex tracer [19]. Thus, in the first stage of transition, the inner and outer shear layers roll up into quasi-axisymmetric vortex rings due to Kelvin-Helmholtz instabilities. For a high velocity ratio coaxial jet $\left(r_{u}>1\right)$, inner and outer vortices turn in opposite sense. Moreover, they undergo a 'locking' phenomenon. Indeed, the inner structures are trapped in the free space between two consecutive outer structures. Therefore, the evolution of the inner vortices is dictated by the motion of the outer vortices. This implies for the inner and outer shear layers identical frequencies corresponding to the theoretical value predicted by the linear-instability theory for the outer shear layer [20]. The appearance of primary structures is characterized by two peaks in the rms axial velocity (noted $\left\langle u^{\prime 2}\right\rangle^{1 / 2}$, where the brackets stand for a temporal averaging calculated by accumulated data over 6000 instantaneous fields over a time period which allows more than

Table 1. Characteristics of the simulations. $M_{1}$ and $M_{2}$ are the initial momentum flux of the inner and outer jet, respectively.

\begin{tabular}{lrcccc}
\hline Name & $r_{u}$ & $R_{1} / \theta_{01}=R_{1} / \theta_{02}$ & $\beta$ & $M_{2} / M_{1}$ & $\begin{array}{c}\text { Jet introducing } \\
\text { species B }\end{array}$ \\
\hline DNS1O & 5 & 12.5 & 2 & 27 & Outer \\
DNS1I & 5 & 12.5 & 2 & 27 & Inner \\
DNS2O & 17 & 12.5 & 2 & 60 & Outer \\
DNS3O & 5 & 25 & 2 & 41 & Outer \\
DNS4O & 5 & 25 & 1.54 & 20 & Outer \\
\hline
\end{tabular}




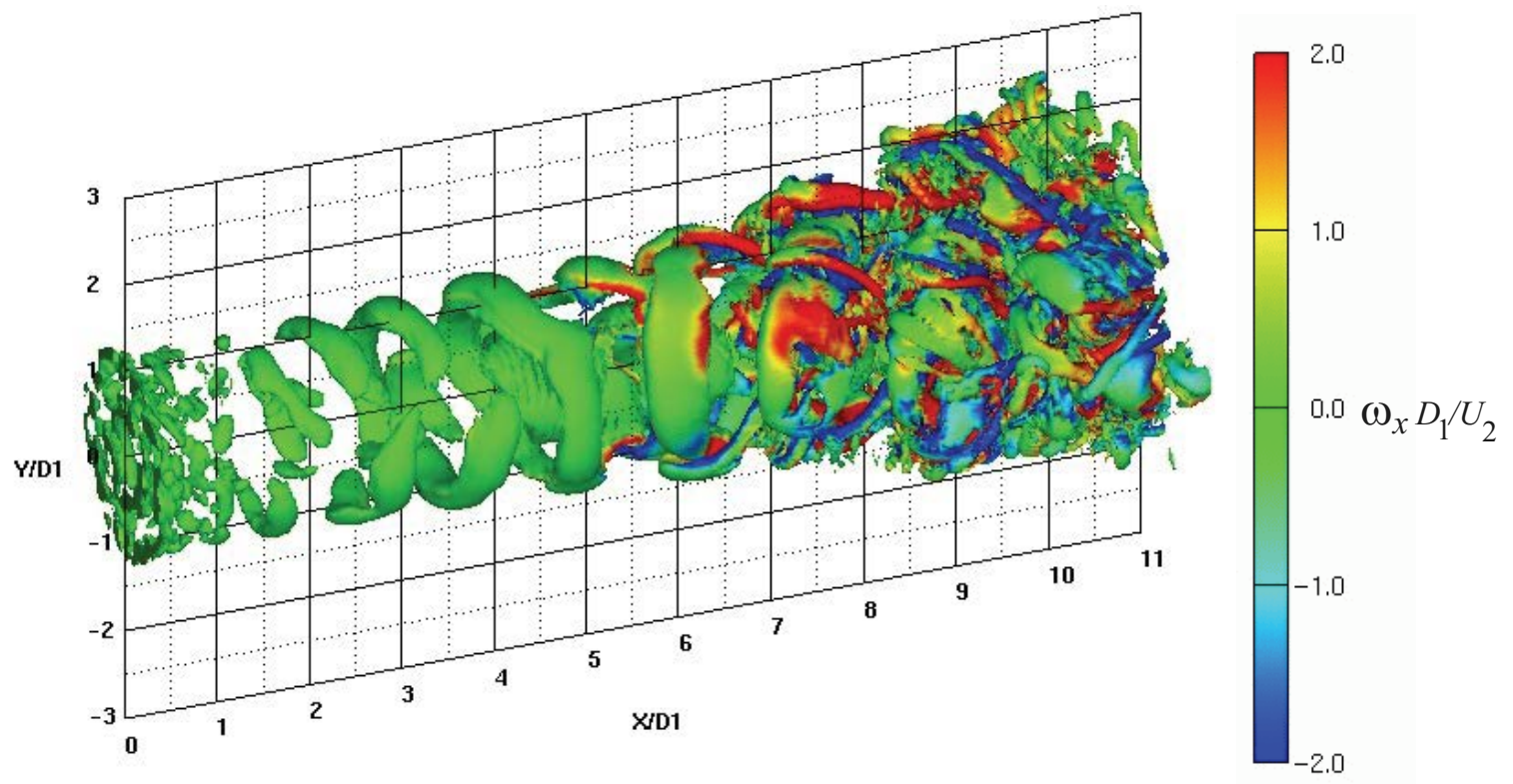

Figure 2. Positive $Q$ isosurfaces $\left(Q=0.5\left(U_{2} / D_{1}\right)^{2}\right)$ colored by the streamwise vorticity $\omega_{x}$ following the color scale shown in the figure, for DNS1O.

30 Kelvin-Helmholtz vortices to leave the computational domain) profiles localized on the inner and outer shear layers as shown by figure 3(b) at locations $x / D_{1}<6$. When $x / D_{1}>6$, there is a rapid spatial growth of rms quantities. This is due to the appearance of pairs of streamwise vortices between two consecutive outer and inner primary vortices (figure 2 ) in agreement with the classical scenario of transition in free shear layers. In a round jet, counterrotating streamwise vortices cause radial ejection of fluid from the core of the jet to its periphery [21]. Note that the location where streamwise vortices appear is very sensitive to upstream conditions [2]. Before this stage, the coaxial jet keeps a mean velocity profile similar to the upstream profile. But for $x / D_{1}>8$, the axial velocity profile resembles more a single round jet (figure 3(a)). Indeed, the profile loses its two-layer structure and the maximum velocity moves toward the centerline [22]. At the end of the computational domain, the most intense turbulent activity is localized in the outer annular jet (figure 3(b)). In this region, there is an abrupt increase in the small-scale turbulence level. The flow reaches a fully developed turbulent state and the time-frequency spectrum of the axial velocity component has a $-5 / 3$ range over about one decade (figure 3(c)). Moreover, there is a strong momentum transfer from the outer annular jet to the inner jet for a high-velocity ratio. This transfer implies a 'pinching' of the central jet by the annular jet [23].
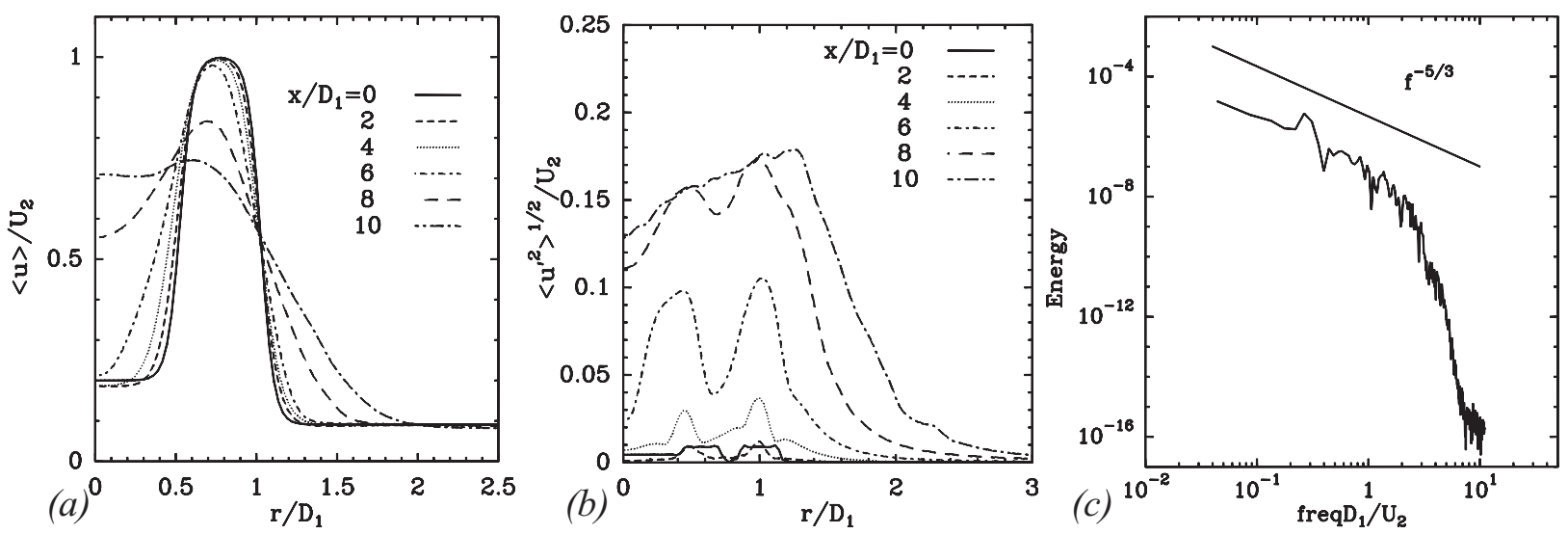

Figure 3. Mean axial velocity profiles (a) and rms axial velocity profiles (b) of DNS1O at several sections. Frequency spectrum of the axial velocity signal for DNS1O in the center of the jet at the end of the computational domain (c). 

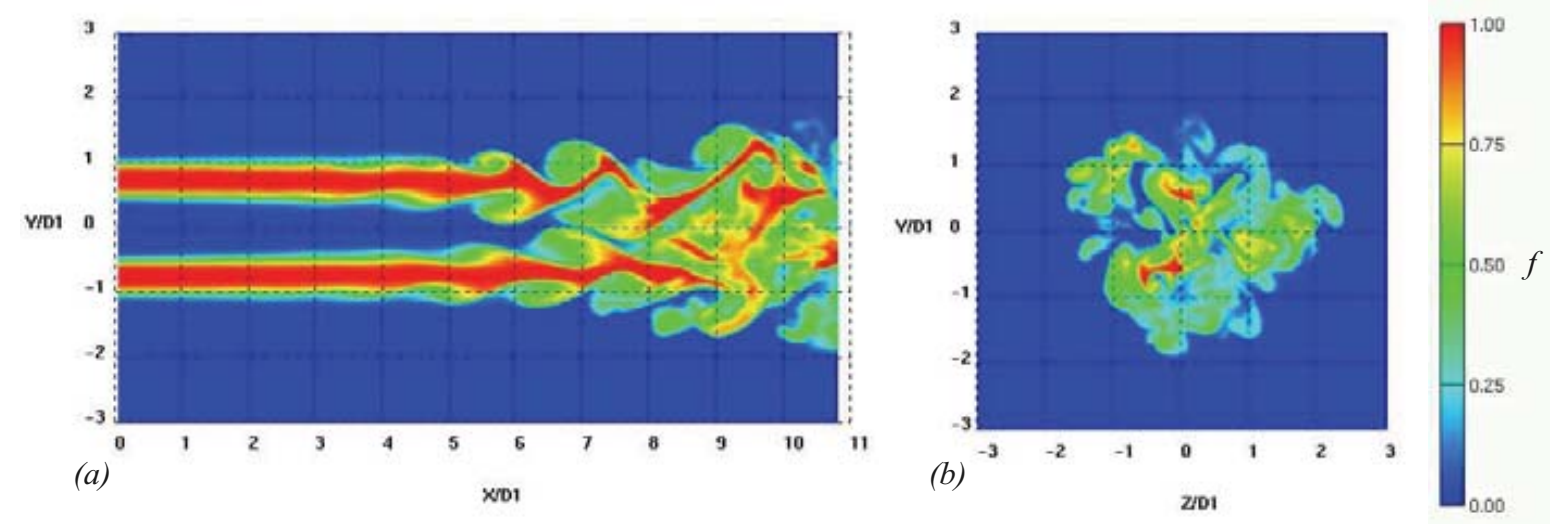

Figure 4. Instantaneous contours of mixture fraction for the DNS1O case: (a) in the central plane and (b) in the transverse section located at $x / D_{1}=10 . f$ varies from 0 (blue) to 1 (red) following the color scale shown in the figure.

\subsection{Species B seeded in the annular jet}

In this case species B is seeded in the annular jet and the remainder of the flow (inner jet and co-flow) contains species A. An inlet mixture fraction profile ensuring $f=1$ in the annular stream and $f=0$ elsewhere is prescribed.

To illustrate mixing qualitatively, the instantaneous planar flow along the central plane is shown in figure 4(a). In the near-field region until $x / D_{1}=5$, the disturbances are weak, in such a way that nonlinear interactions are insignificant. Thus, species exchange between annular and central jets and between the annular jet and the co-flow are mainly dominated by molecular diffusion in this region. Note that the extension of this region is important because a white-noise perturbation with a weak amplitude is used to allow the development of the 'natural' instabilities and this implies that the initial evolution of the shear layers is very smooth [1]. After $x / D_{1}=5$, a turbulent mixing activity appears because of coherent vortices. Finally, at the end of the computational domain $\left(x / D_{1}>10\right)$, turbulent small scales allow an intense turbulent mixing as shown by figure 4(b). Figure 5 shows the mixture fraction $f=0.5$ isosurface. It is a good indicator of mixing efficiency in turbulent mixing conditions.

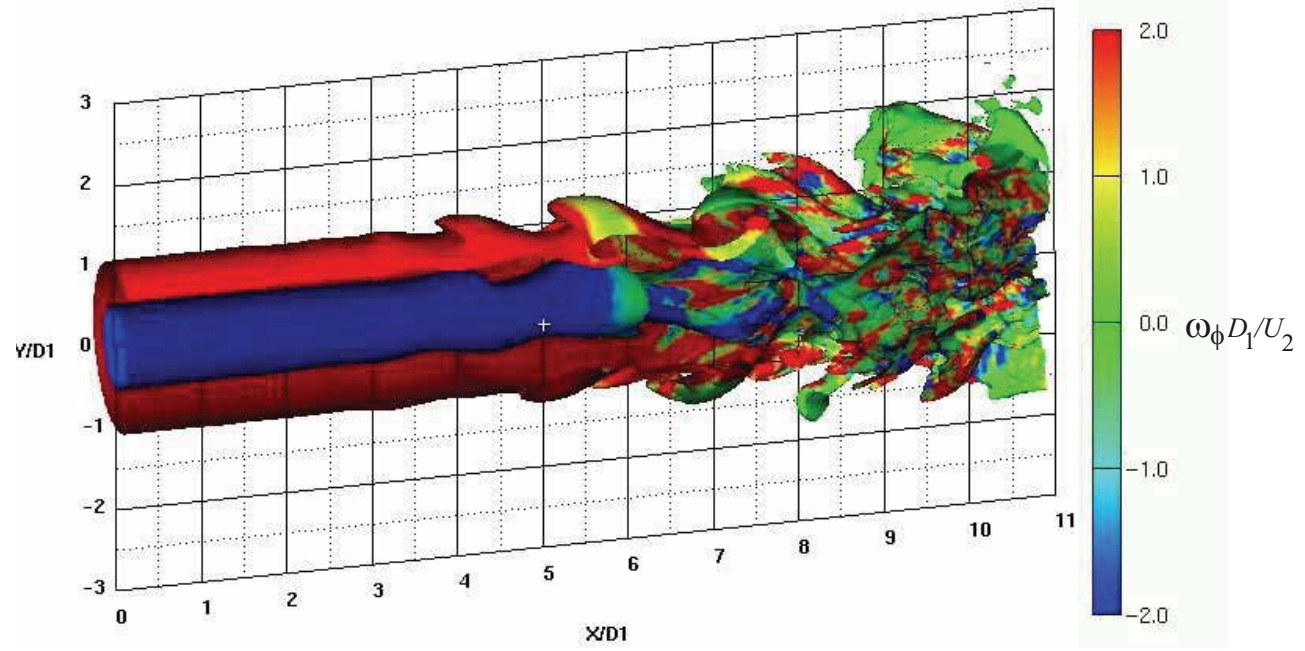

Figure 5. Cut view of $f=0.5$ mixture-fraction isosurface colored by the tangential vorticity $\omega_{\phi}$ following the color scale shown in the figure, for DNS1O. 


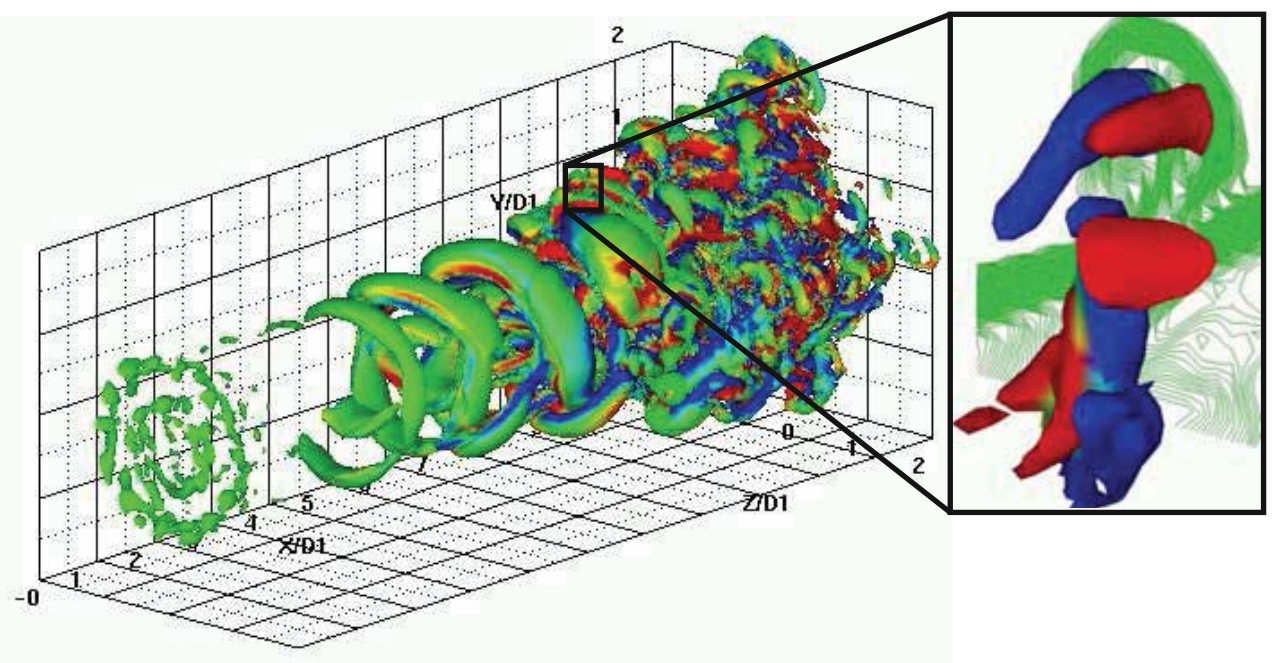

Figure 6. Illustration of passive scalar ejections (shown by green contours on a zoom) by the counter-rotating longitudinal vortices (shown by an isosurface of $Q=0.7\left(U_{2} / D_{1}\right)^{2}$ and colored in red or blue according to the longitudinal-vorticity sign as used in figure 2).

Characterization of the multi-scale geometry of the iso-species surfaces is a crucial step for understanding and modeling turbulent mixing linked to turbulent combustion processes. The $f=0.5$ isosurface is highly convoluted after the instabilities amplification, with evidence of a large-scale structure. Thus, the inner and outer Kelvin-Helmholtz vortices allow for an engulfment of the two fluid streams placed on each side of the inner and outer shear layers, respectively. This phenomenon initiates the turbulent mixing. In figure 5 the isosurface is colored by the azimuthal vorticity field, $\omega_{\phi}$ defined in the cylindrical coordinate system $(x, r, \phi)$, to distinguish the inner and the outer mixing layers (as used previously by da Silva et al. [1] to study coherent vortices). One can distinguish two notable shapes of the isosurface: before $x / D_{1} \approx 7$, the two parts of the isosurface (for the outer and the inner mixing layers) are wholly distinct, whereas for $x / D_{1}>7$ they are strongly merged, showing inter-penetration between species A and B. This merging between both mixing layers is a consequence of the action of streamwise vortices. Indeed, these counter-rotating vortices allow us to eject species $\mathrm{B}$ into the ambient fluid as shown by figure 6 . An exhaustive observation of an animation (see Animation_1) showing the $f=0.5$ isosurface and planar visualization in $y-z$ plane reveals that the intermittent specificity of the turbulent process combines a radial periodic pulsation of the ring structures with intermittent mushroom-type structures characterizing the ejection phenomenon due to the longitudinal counter-rotating vortices. Moreover, large amounts of unmixed species B persist as spots even far downstream where stirring conditions are strong and the diffusion interface is enlarged (figure 4). An appropriate frequency forcing of 3D longitudinal structures as well as of vortical rings at the inlet boundary could decrease the amount of pure species [24].

Figure 7 shows the evolution of the mean mixture fraction in the downstream direction (a) and at several sections of the jet (b). For $x / D_{1}<5$, one can observe on the axial development of the mixing fraction in both the inner and the outer jets that $\langle f\rangle$ remains equal to 0 and 1 , respectively. The radial mean mixture fraction profiles at various downstream sections indicate clearly for $x / D_{1}<5$ a variation of $\langle f\rangle$ at the interface between the two species $\mathrm{A}$ and $\mathrm{B}$. This variation is due to the molecular diffusion which mixes A and B in the radial direction. Downstream of $x / D_{1}=5$, there is an important invasion of species B into the central jet. This invasion allowed by the momentum transfer already discussed above implies that the fluid issued from the central jet is confined (see the DNS1I case). Moreover, species B of 

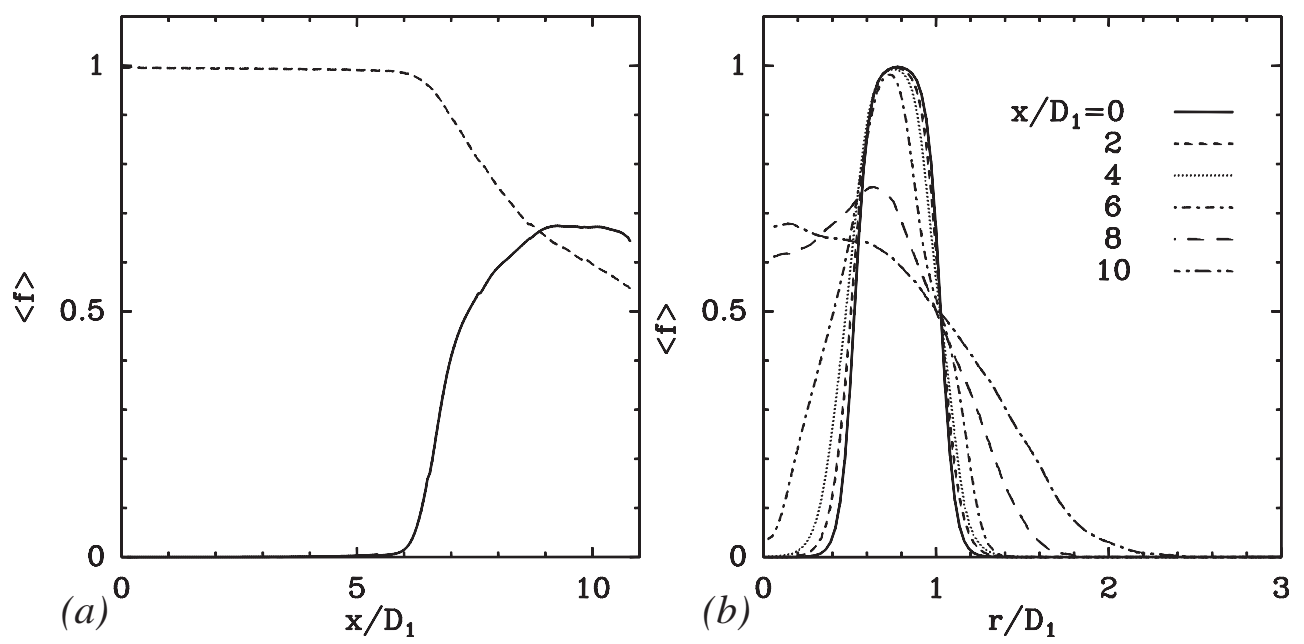

Figure 7. Mean mixture fraction. (a) Downstream evolution in both central (continuous line) and annular jets (dashed line). (b) Radial evolution at several downstream locations.

the annular jet diffuses appreciably in the co-flow at these downstream locations. Figure 7(a) shows that the entrainment of species into the shear layers improves the mixing activity beyond $x / D_{1}=5$. The mean mixture fraction, respectively, decreases in the annular jet and increases in the central jet, to reach an asymptotic value around $\langle f\rangle=0.5$. The increase of the mixture fraction in the inner jet is more important than the decrease of the mixture fraction in the outer jet. Hence, there is a larger amount of species B invading the inner jet than species A invading the outer jet. The mixture fraction rms is displayed in figure 8. It confirms that the turbulent mixing becomes significant downstream of $x / D_{1} \approx 5$. This is due to longitudinal coherent vortices which allow us to eject species B and bring three dimensionality to the jet. Note that the peak of the inner jet is the location where species B invades the center and there is an important turbulent mixing activity in this region. But upstream of $x / D_{1} \approx 5$, the radial profiles at various axial positions (figure 8(b)) indicate two distinct peaks located in the inner and outer shear layers, respectively. The two peaks are the signatures of the large structures which initiate the turbulent mixing. In the near field, the mixture-fraction frequency computed in the inner and outer shear layers are equal (figure 9) because of the 'locking' phenomenon already discussed. For $x / D_{1}>8$, figure 7(b) shows that the mean mixture fraction is maximum in the jet center. Close to the exit, large amounts of species B invade the central jet and the inner and outer mixing layers merge in order to create a single-mixing zone similar to a single jet. When looking at the mixture-fraction rms profiles (figure $8(\mathrm{~b})$ ), it is only the outer
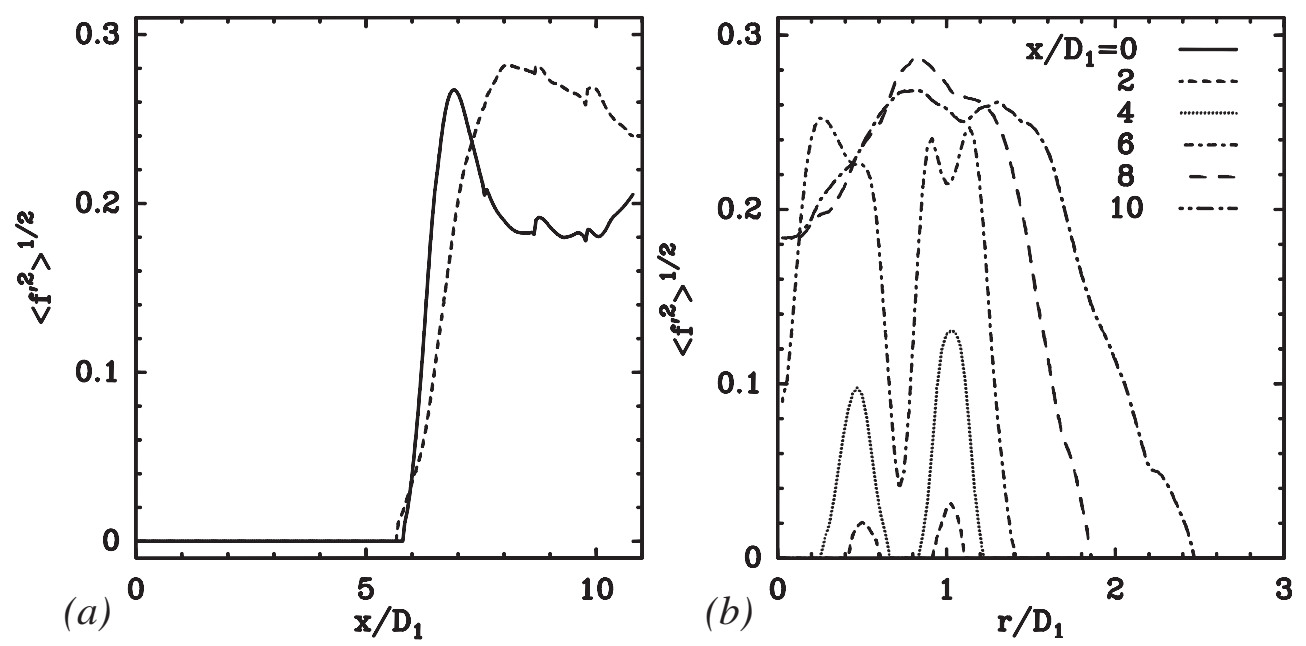

Figure 8. Rms of the mixture fraction. (a) Downstream evolution in both central (continuous line) and annular jets (dashed line). (b) Radial evolution at several downstream locations. 


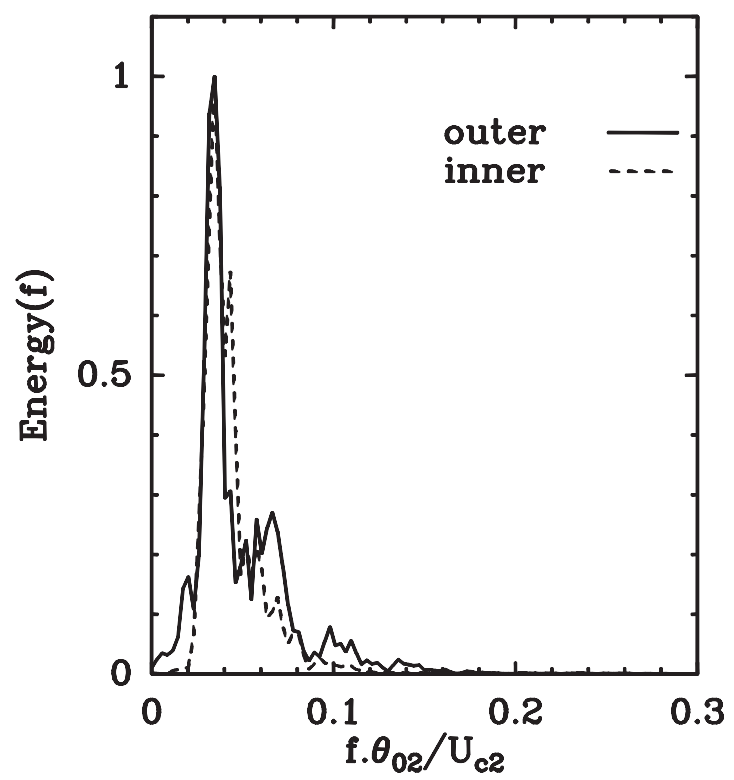

Figure 9. Frequency spectra of the mixture fraction signal for DNS1O in the near field of the jet at the inner and outer shear layers. $U_{c 2}$ is the velocity averaged across the outer shear layer and $\theta_{02}$ the outer momentum thickness. Energy is normalized by its maximum value.

peak which persists in this region. It is a consequence of the outer-jet dominance, as far as mixing properties are concerned.

Now, in order to characterize the development of the mixing process, it is of interest to study the variations of the probability density functions at various axial and radial positions [25]. This information is useful in the development of models to describe mixing, especially for combustion issues. The mixture fraction PDF at three streamwise locations covering the transition stages are displayed in the three following figures. Figure 10 shows the PDF in both the inner and outer jets at the upstream position $x / D_{1}=2$. It is obvious from the shape of the profiles that the mixing behavior is solely due to molecular diffusion, since stirring and turbulent mixing are not at hand in the early flow development $\left(x / D_{1}<5\right)$. This is essential to interpret simulations presented below with regard to the effect of various inlet conditions on the mixing activity of the whole field. In figure 11, the mixture-fraction PDF are shown at $x / D_{1}=5$, preceding the self-similar state. They reveal the existence of a broad range where the probability of finding a given value of the mixing fraction is not weak for each
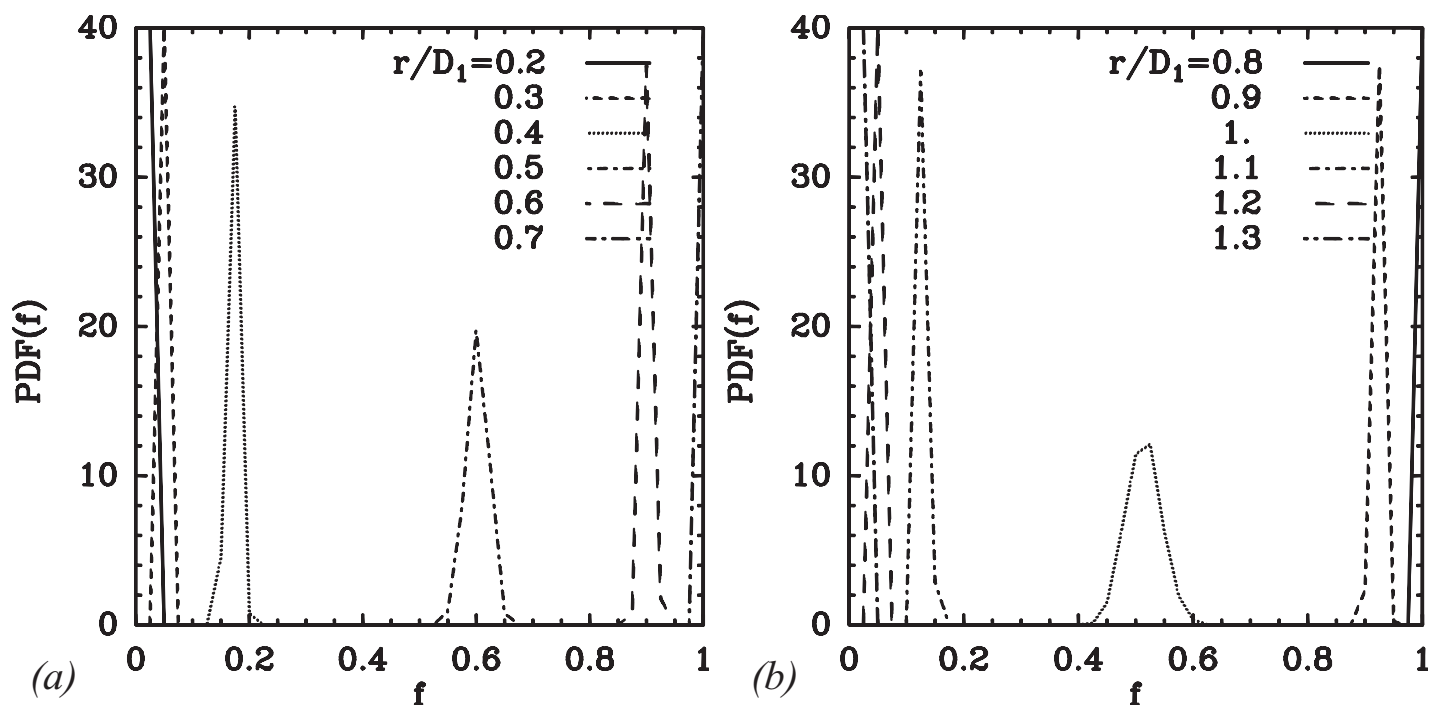

Figure 10. Variation of the mixture fraction PDF across the inner (a) and outer (b) mixing layer at $x / D_{1}=2$. 

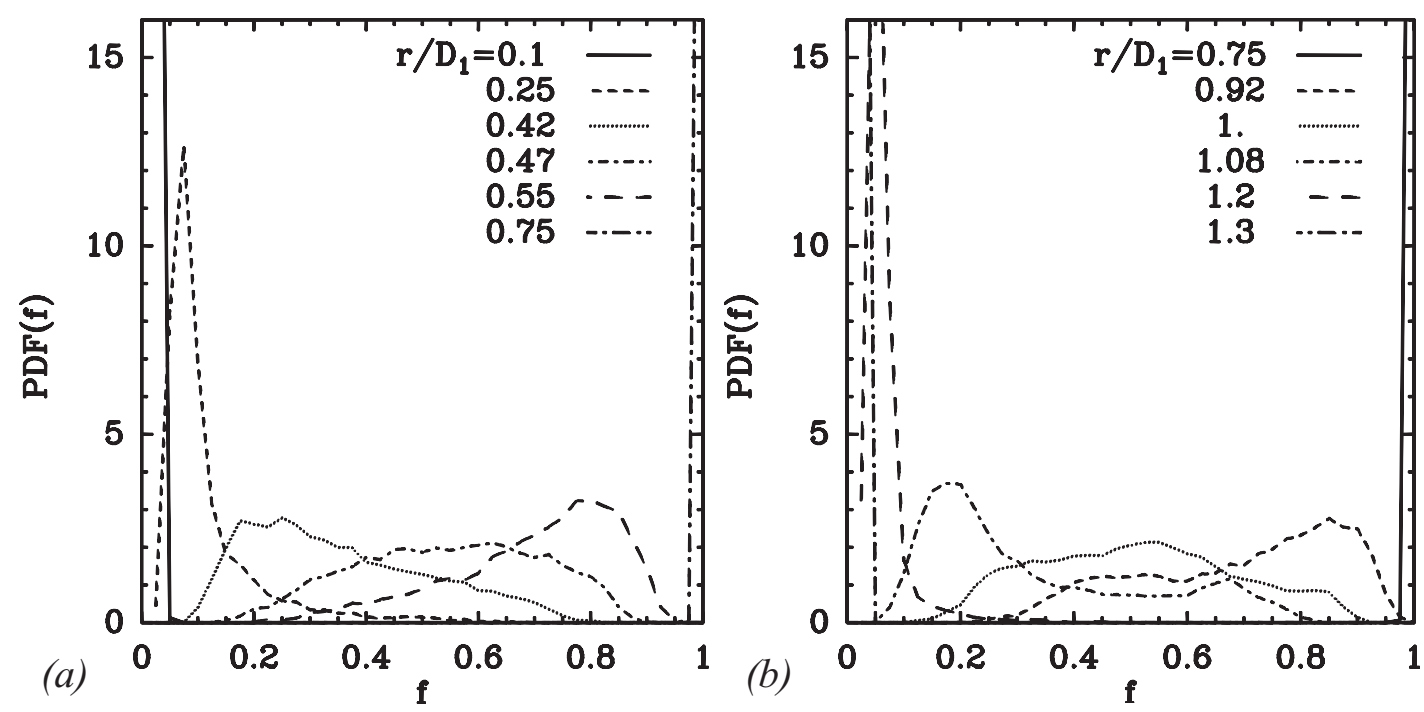

Figure 11. Variation of the mixture fraction PDF across the inner (a) and outer (b) mixing layer at $x / D_{1}=5$.

radial position $r$. This is due to the periodicity of the turbulent mixing process that is linked to the frequency at which Kelvin-Helmholtz coherent vortices appear within the two shear regions. Thus at this downstream location, the mixing between species A and B is then not quite homogeneously distributed inside the large-scale structures, prior to turbulent transition. Furthermore, a marching feature is discernible for both the inner and the outer jets. PDFs are called marching if the most probable value of $f$ varies across the layer and equals the free stream value of each side of the layer. They are called non-marching if the most probable value of $f$ is notably independent of the location within the layer. In the middle of the mixing layers (i.e. the points $r / D_{1}=0.47$ for the inner mixing layer and $r / D_{1}=1$ for the outer mixing layer), the most probable value is $f \approx 0.5$. The A-B mixing therefore occurs in equal proportion at these two radial locations. Furthermore, the trace of unmixed species B is clearly detectable in figure 11 (b) for $r / D_{1}=0.75$ with the high plausible value $f \approx 1$. Figure 12 illustrates the mixture-fraction PDF at $x / D_{1}=10$. At this downstream location the inner and outer mixing layers have merged into a single-mixing layer, with the domination of the outer mixing layer. A further examination reveals a non-marching PDF for the mixing layer, as the most probable value of $f$ is quasi-independent of the radial positions, and is not the mean value of $f$. Moreover, pure unmixed species B is also perceptible at this streamwise

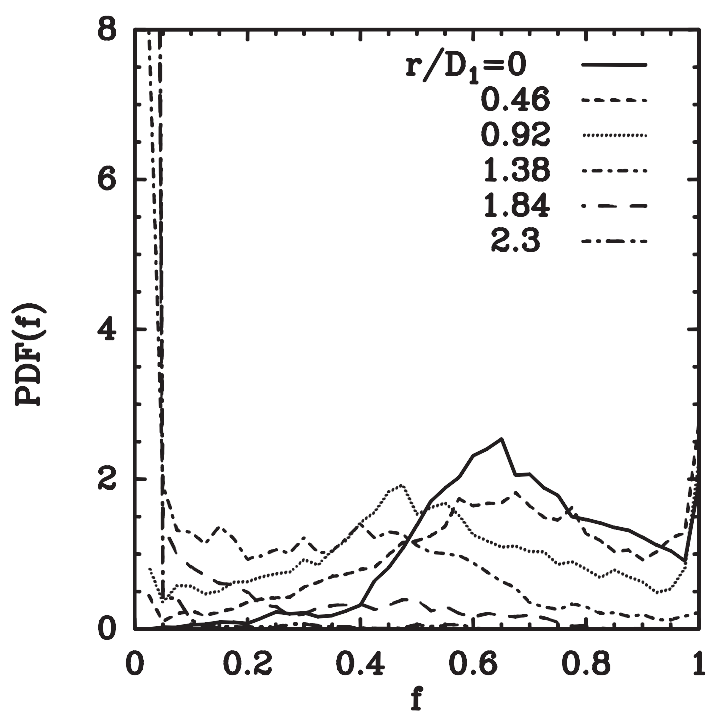

Figure 12. Variation of the mixture fraction PDF across the mixing layer at $x / D_{1}=10$. 

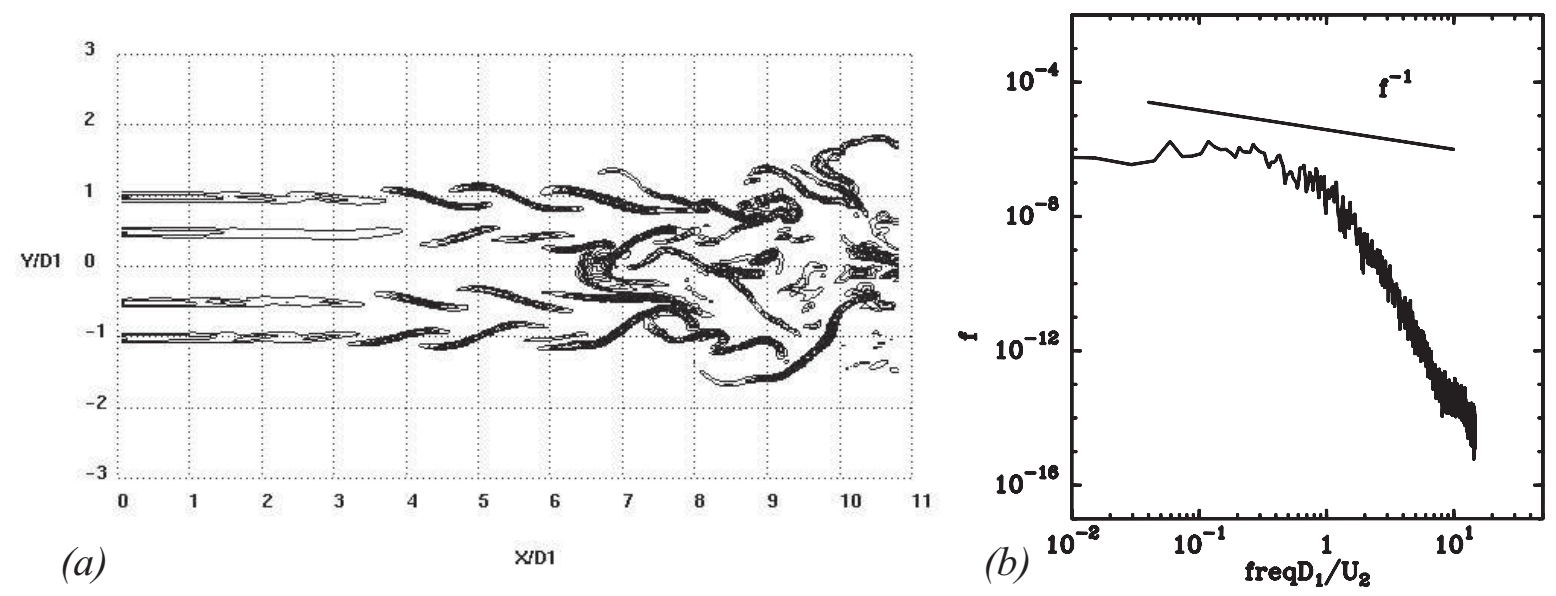

Figure 13. (a) Instantaneous mixture fraction dissipation field in the central plane. (b) Time-frequency spectrum of the mixture fraction at $x / D_{1} \approx 10$.

location for $r / D_{1}=0,0.46$ and 0.92 . These facts show that the turbulent mixing activity is important and that the mixing is not again homogeneous in this region where the turbulent flow has reached a fully developed state.

Figure 13(a) shows the instantaneous scalar dissipation field, whose analysis is of great importance for scalar subgrid modeling in large-eddy simulations of reacting flows. The intense scalar dissipation regions have a sheet-like structure and are located at the boundaries of the large coherent vortices as seen in turbulent shear flows by Buch and Dahm [26]. This confirms the very intermittent nature of the passive scalar as previously observed by Métais and Lesieur [27] in isotropic turbulence. Métais and Lesieur [27] showed that the scalar activity is indeed concentrated on braids between the coherent vortices in agreement with the present observations. Lesieur and Rogallo [28] and Métais and Lesieur [27] furthermore observed that this scalar intermittency is associated with an anomalous spectral slope following a $k^{-1}$ law ( $k$ wavenumber) for the scalar variance. Lesieur and Rogallo [28] propose that the spectral behavior is a result of the shearing by large-scale velocity gradients. Let us assume that the large scales are associated with a given wavenumber $k_{i}$. Assuming that the scalar variance flux $\epsilon_{f}$ is independent of $k$ and using the Oboukhov-type theory, Lesieur and Rogallo [28] write

$$
\epsilon_{f} \sim k E_{f}(k, t) / \tau\left(k_{i}\right)
$$

In equation (3), $E_{f}(k, t)$ is the scalar variance spectrum and $\tau\left(k_{i}\right)$ is the turnover time of $k_{i}$. Thus, from equation (3), they find

$$
E_{f}(k, t) \sim \epsilon_{f} \tau\left(k_{i}\right) k^{-1}
$$

Lesieur and Rogallo [28] observe a $k^{-1}$ range in the spectral neighborhood of $k_{i}$ for $k>k_{i}$ in very good correspondence with the theoretical prediction given by equation (4). This behavior is confirmed by figure 13(b) which shows the time frequency spectrum for the mixture fraction: it exhibits a slope close to $f^{-1}$. Note that Villermaux and Rehab [5] also found a $k^{-1}$ shape on the spectra of scalar fluctuations of coaxial jets which they explained by the persistence of the large-scale straining motion. These points however require further analysis which will be the object of a further study. 


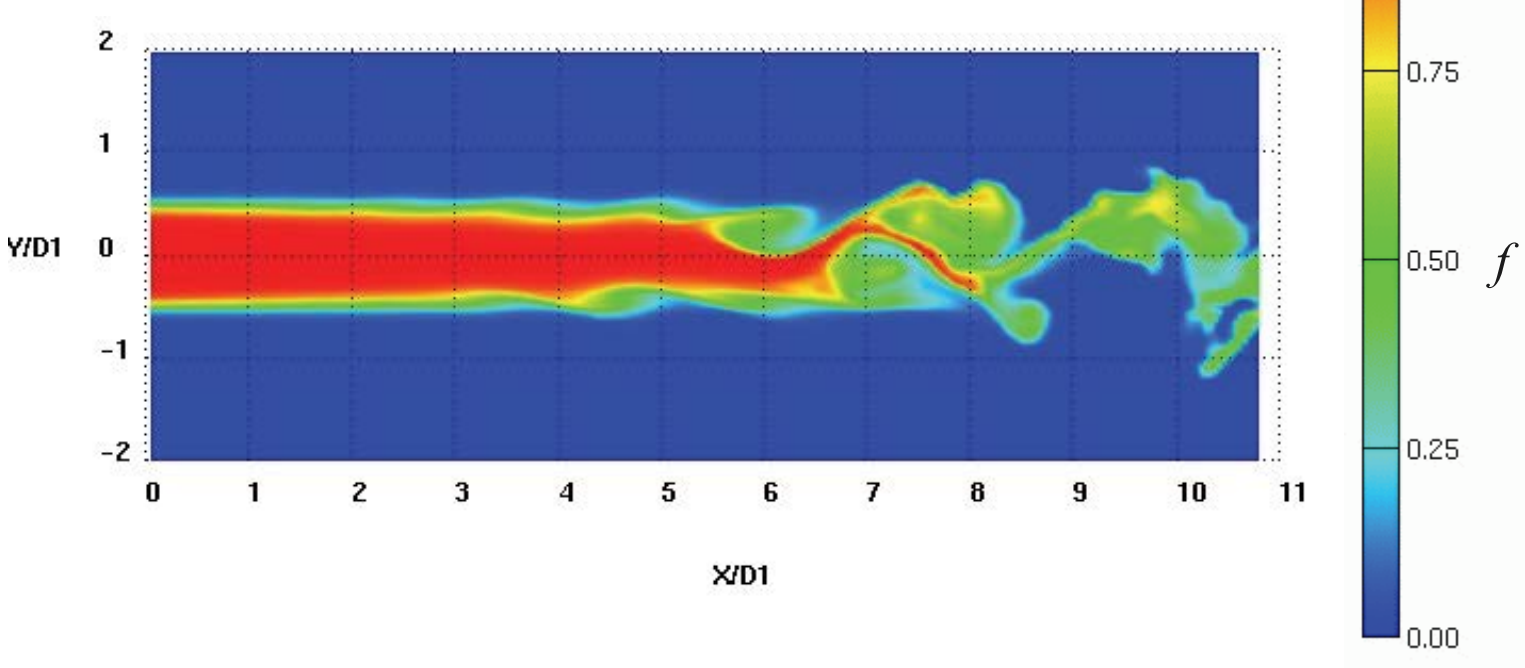

Figure 14. Instantaneous contours of mixture fraction for the DNS1I case in the central plane. $f$ varies from 0 (blue) to 1 (red) following the color scale shown on the figure.

\section{Influence of the upstream conditions on the mixing process}

\subsection{Species $B$ seeded in the central jet}

We study now the alteration on the mixing process if species B is seeded in the low-speed central jet and the remainder of the flow (annular jet and co-flow) contains species A. The coaxial jet keeps the dynamical parameters of DNS1O. This mixing configuration is called DNS1I. We prescribe an inlet mixture-fraction profile which ensures a value of $f=1$ in the central jet and $f=0$ elsewhere.

Figure 14 gives a global picture of the mixing for DNS1I. First, there is an important initial region dominated by molecular diffusion. The appearance of Kelvin-Helmholtz vortices induces the beginning of turbulent mixing. Note that in this configuration, only the inner vortices participate in the mixing. Indeed, the outer vortices are only surrounded by species A $(f=0)$. This stage is followed by the emergence of the streamwise vortices which eject species in mushroom-type structures. This point appears clearly in the animation (see Animation_2) of the $y-z$ planes. However, the comparison between figures 4(a) and 14 shows a confinement of species B in the jet center for DNS1I. Figure 15 shows the $f=0.5$ isosurface of the mixture fraction. It reveals the important difference of the mixing process between DNS1I and DNS1O beyond $x / D_{1}=6$. Indeed, while the isosurface is highly convoluted after the amplification of instabilities in this case, the volumetric aspect of the mixing region is much smaller, due to an important confinement. The animation reveals that at the end of the computational domain the scalar passes by packets. This is a consequence of the domination of the outer vortices which do not contribute to the mixing, because they do not transport species B and hinder the radial development of the inner turbulent structures.

The qualitative observations based on visualizations are also supported by DNS1I statistics. Indeed, figures 16(a) and (b) show the downstream evolution of the mean mixture fraction in the central and the annular jets, as well as its radial evolution at several locations. In the central jet, it keeps a constant value $(f=1)$ before $x / D_{1}=6$ and undergoes an important decrease after, while in the annular jet it has a small value in all the computational domain (figure 16(a)). The radial mean mixture fraction profiles at various downstream locations 


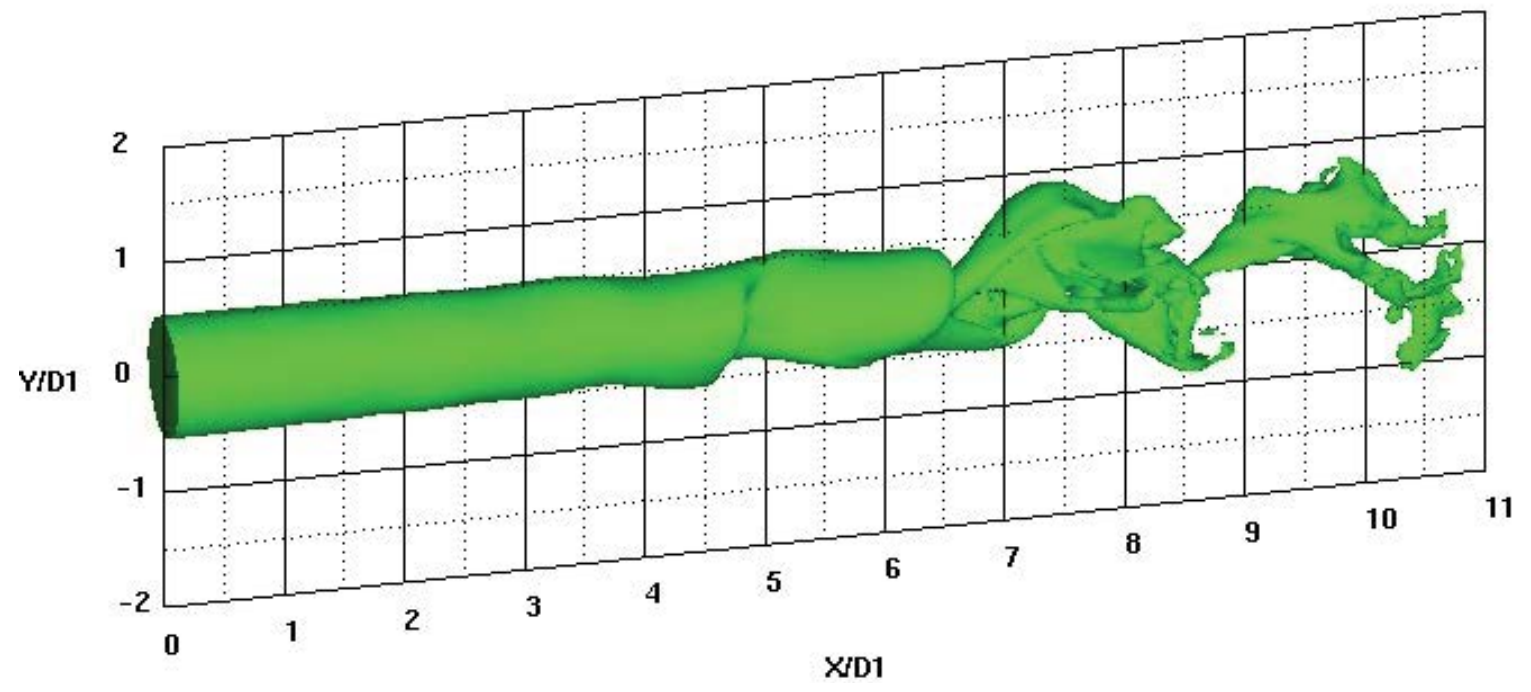

Figure 15. $f=0.5$ isosurface of mixture fraction for DNS1I.

(figure 16(b)) indicates obviously that the maximum variation of $f$ occurs inside the inlet curve for $0<r / D_{1}<1$. This interval corresponds to the edge of the inner jet, and one can observe that a very small amount of species B reaches the annular jet. The mixing cannot take advantage of the important turbulent activity localized in the annular jet because species B does not invade the annular jet (figure 3(b)). Thus, at the end of the computational domain, the mixture rms peak remains localized in the central jet and its intensity (figure 17(b)) is smaller than that of the DNS1O localized in the annular jet (figure 8(b)).

Similarly to the previous case (species B seeded in the annular jet), we examine now PDFs of $f$ at the same downstream locations. Considering that the kinematic inlet conditions are unchanged, it is not useful to plot the PDFs for $x / D_{1}<5$. Figure 18(a) shows the PDFs at $x / D_{1}=5$ prior to the establishment of self-similarity. As in the previous case, in the middle of the inner shear layer $\left(r / D_{1}=0.44\right)$, the probability of finding a given value of $f$ in the range situated between 0.2 and 0.7 is practically the same. The mixing of species A and B is then inhomogenously scattered in the inner vortices. At $r / D_{1}=0.25$, the maximum is close to $f=1$, and species A has not invaded the jet center, since the Kelvin-Helmholtz vortices are not sufficiently developed to reach a self-similar state. One can also observe the absence of species B beyond $r / D_{1}=0.75$. This result indicates that species B initially seeded in the inner
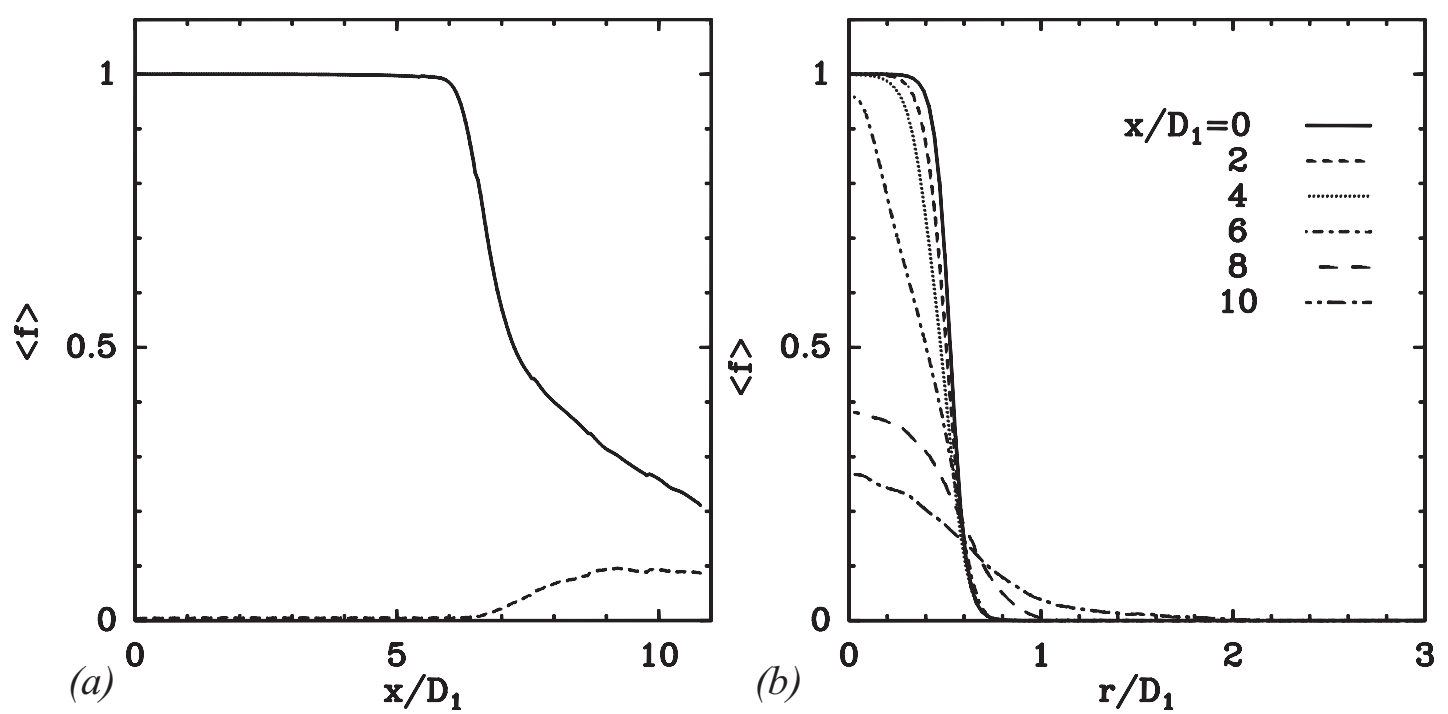

Figure 16. Mean mixture fraction. (a) Downstream evolution in both central (continuous line) and annular jet (dashed line). (b) Radial evolution at several downstream locations. 

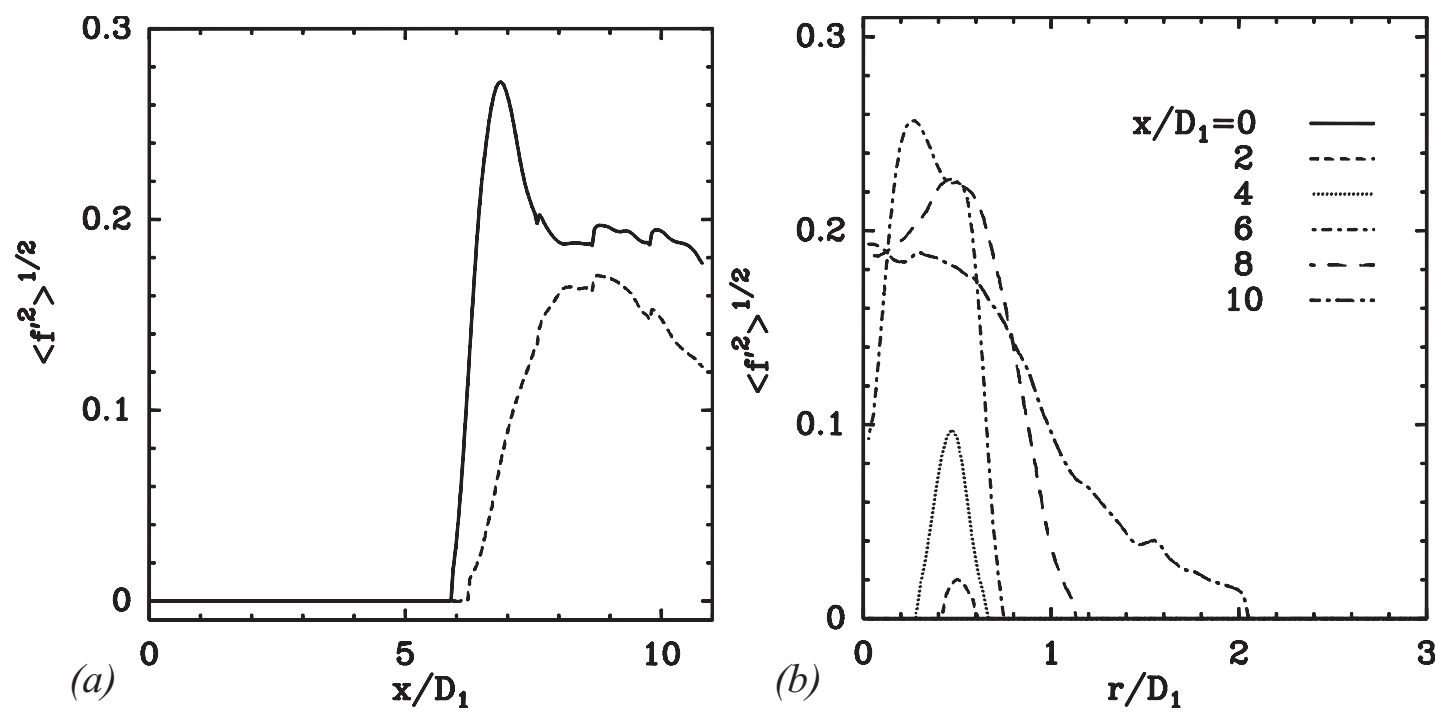

Figure 17. Rms of the mixture fraction. (a) Downstream evolution in both central (continuous line) and annular jets (dashed line). (b) Radial evolution at several downstream locations.

jet (in the case of DNS1I) remains confined between the edge of the inner-outer jets and the centerline. At $x / D_{1}=10$ (figure 18(b)), the PDFs show an important probability of finding a mixture-fraction value in the range $0.2-0.4$ for radial positions near the jet center (until $\left.r / D_{1}=0.5\right)$. Additionally, it is interesting to note that the probability of finding $f>0.7$ or unmixed species $\mathrm{B}(f=1)$ for all the radial positions is zero. Thus, all the amount of species $\mathrm{B}$ initially released in the inner jet is totally mixed with species A, especially just beyond the turbulent transition.

\subsection{Velocity ratio and recirculation bubble}

When the velocity ratio $r_{u}$ increases, the entrainment by the outer annular jet and the depletion of the inner fluid is more and more pronounced. If $r_{u}$ exceeds a critical value $r_{u c}$, the inner potential core breaks down and a back-flow region establishes associated with a negative streamwise velocity around the jet center. The recirculation bubble has been studied experimentally and numerically in previous works $[2,23]$. The recirculation bubble is a region where there is a significant production of streamwise vortices. We investigate in this section the influence of the recirculation bubble on the mixing process. The simulation
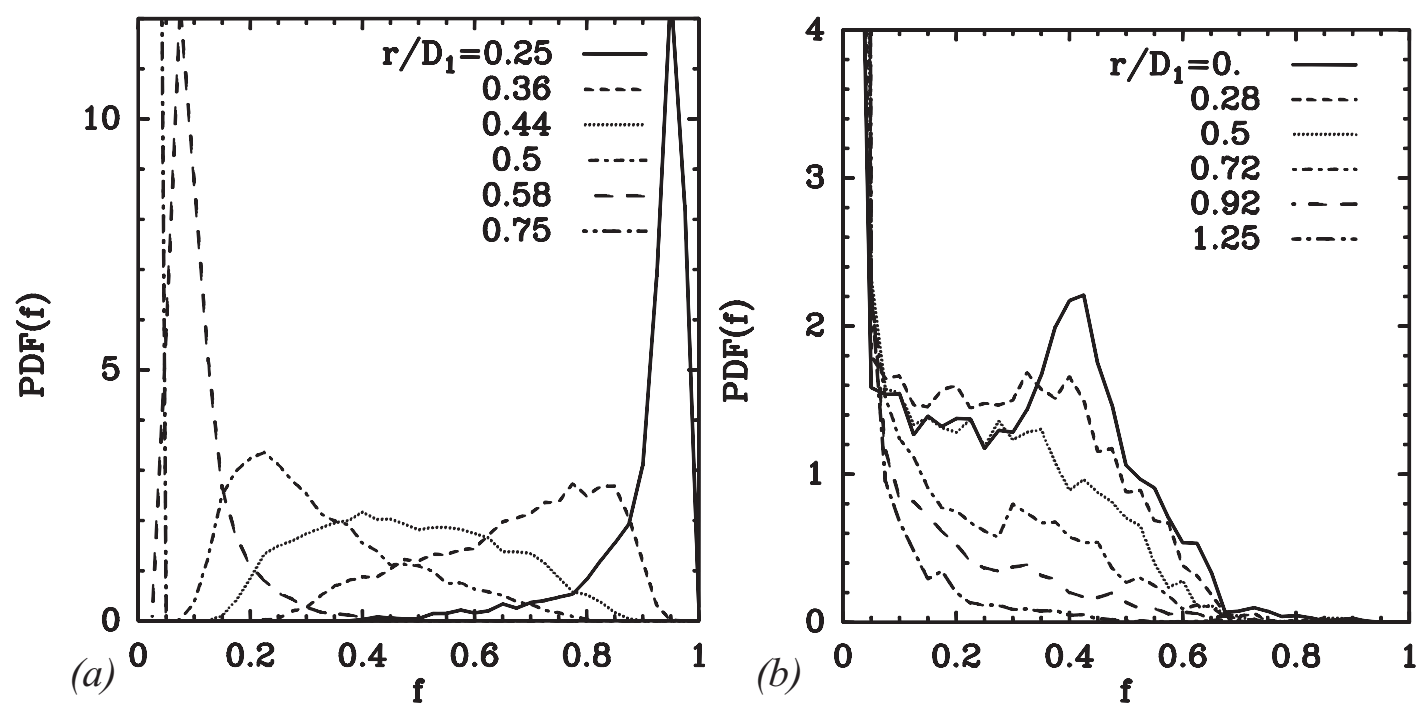

Figure 18. The variation of the mixture fraction PDFs across the mixing layer at $x / D_{1}=5$ (a) and $x / D_{1}=10$ (b). 


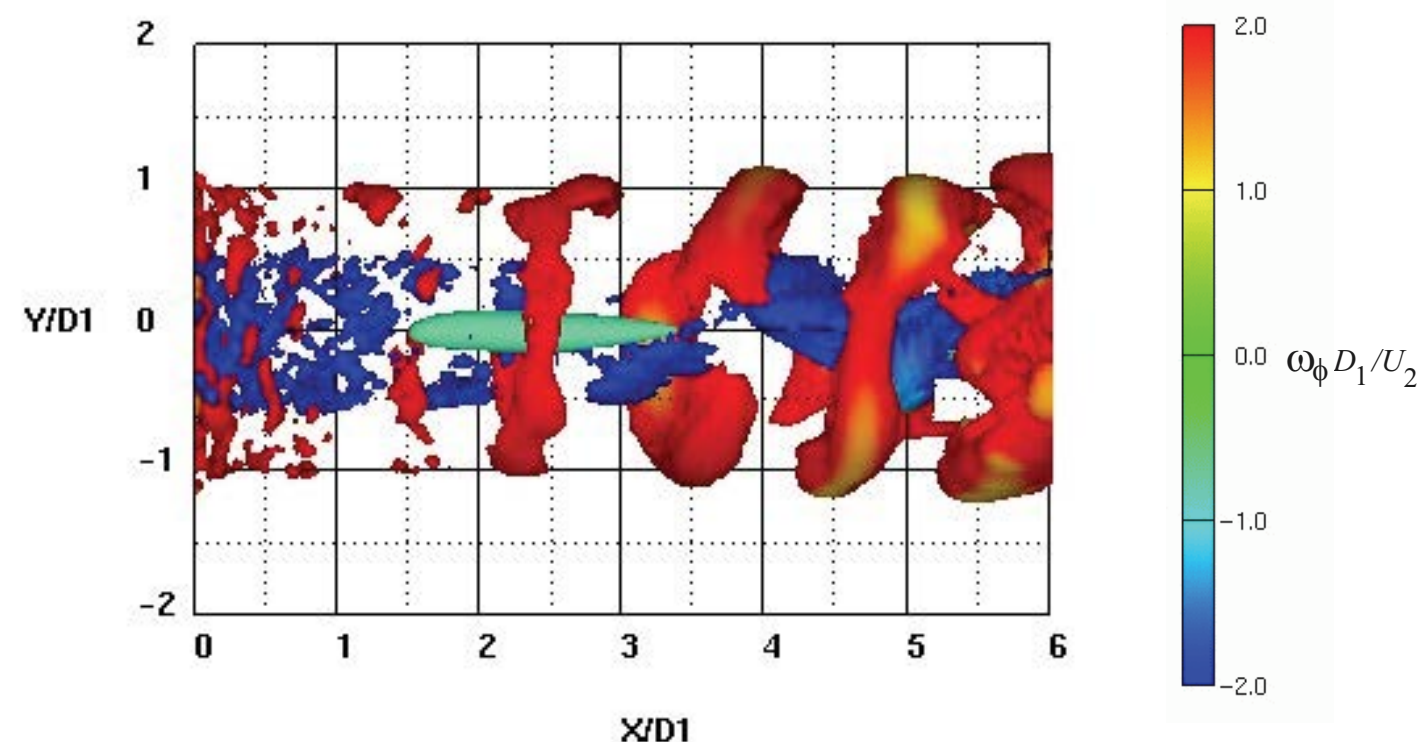

Figure 19. Positive $Q$ isosurfaces $\left(Q=0.5\left(U_{2} / D_{1}\right)^{2}\right)$ colored by the tangential vorticity $\omega_{\phi}$ following the color scale shown in the figure, for DNS2O. Isosurface of zero velocity in cyan shows the recirculation bubble.

DNS2O is performed with the same parameters as DNS1O except for the velocity ratio $r_{u}=17$ (with these initial conditions, $r_{u c} \approx 13$ [2]). With this upstream condition DNS2O has a recirculation bubble in the jet center localized between $x / D_{1} \approx 1.5$ and $x / D_{1} \approx 3.5$ (figure 19).

Figure 20 shows instantaneous mixture-fraction isolines in the central plane. The first consequences of the recirculation bubble on the mixing are obviously observable. Species B invades the jet center in the early flow development, e.g. $x / D_{1} \approx 1.5$, whereas in DNS1O case, the jet center is devoid of species B until $x / D_{1} \approx 6$. This upshot is due to the back flow issued from the annular jet, which is responsible for the migration of species $B$ in the central jet at this downstream location. Figure 21 shows the $f=0.5$ isosurface, which is highly convoluted by the large-scale vortices, as shown in the preceding cases. The two isosurfaces (corresponding to the inner and the outer mixing layers, respectively) are wholly decorrelated in the near field of the jet, but the inner isosurface section begins to decrease at $x / D_{1} \approx 3$. This is due

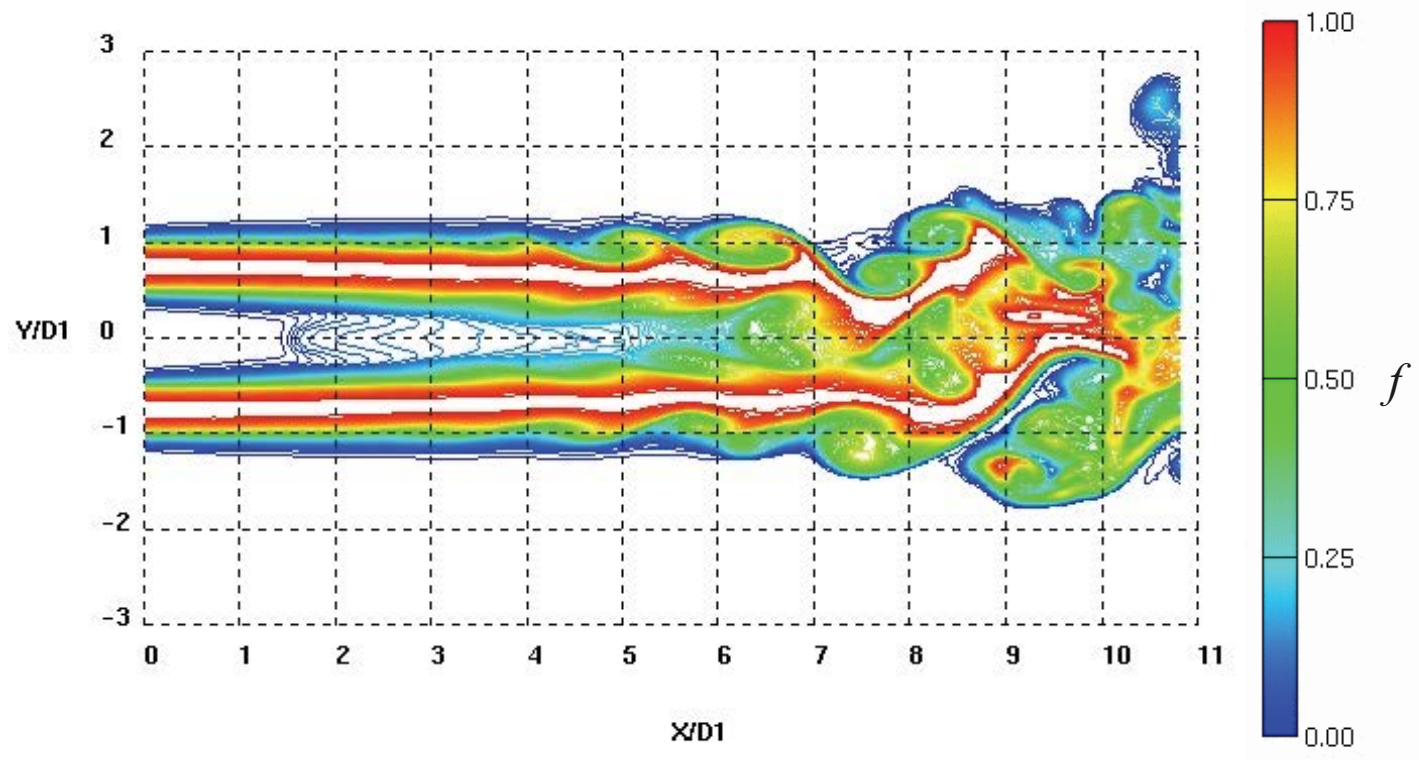

Figure 20. Instantaneous isolines of mixture fraction for the DNS2O case in the central plane. $f$ varies from 0 (blue) to 1 (red) following the color scale shown on the figure. 


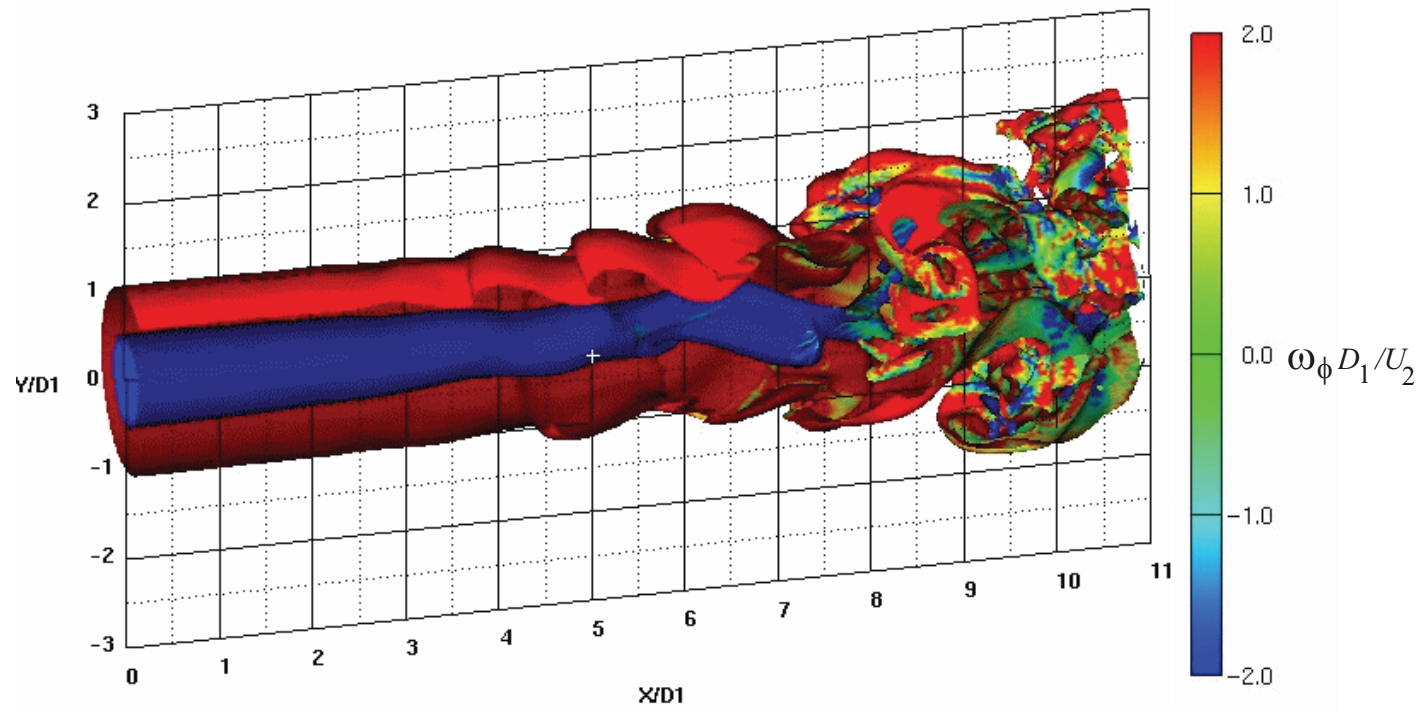

Figure 21. Cut view of $f=0.5$ mixture-fraction isosurface colored by the tangential vorticity $\omega_{\phi}$ following the color scale shown on the figure, for DNS2O.

to a 'pinching' phenomenon which increases for high values of the velocity ratio. The inner isosurface shape is more conical. It is implied by the strong momentum transfer existing for very high velocity ratio coaxial jets [1].

The presence of species $\mathrm{B}$ within the jet center at $x / D_{1} \approx 1.5$ is also confirmed by the statistics of $f$. Indeed, the profile of $\langle f\rangle$ on the jet axis (figure 22(a)) indicates obviously that $\langle f\rangle$ varies between 0 and 0.15 for $1.4<x / D_{1}<5$. Furthermore, the radial profile of $\langle f\rangle$ at $x / D_{1}=2$ (figure 22(b)), shows that $\langle f\rangle=0.1$ at the jet center. Another effect of the recirculation bubble is the spreading of species $\mathrm{B}$ until $r / D_{1} \approx 2.8$ at $x / D_{1}=10$ (figure 22(b)) instead of $r / D_{1} \approx 2.2$ in DNS1O. This interesting result is undoubtedly due to the recirculation bubble. Indeed, as demonstrated previously [2], the tip of the recirculation bubble is a region with a significant production of intense streamwise vortices. The precocious development of inner streamwise vortices induces an earlier development of the outer streamwise vortices and consequently enhances the turbulent mixing activity. In fact, the mixture fraction fluctuations $\left\langle f^{\prime 2}\right\rangle^{1 / 2}$ (linked to turbulent mixing) begins to grow earlier at $x / D_{1} \approx 4.5$ in the DNS2O case, instead of $x / D_{1} \approx 6$ for the DNS1O case without
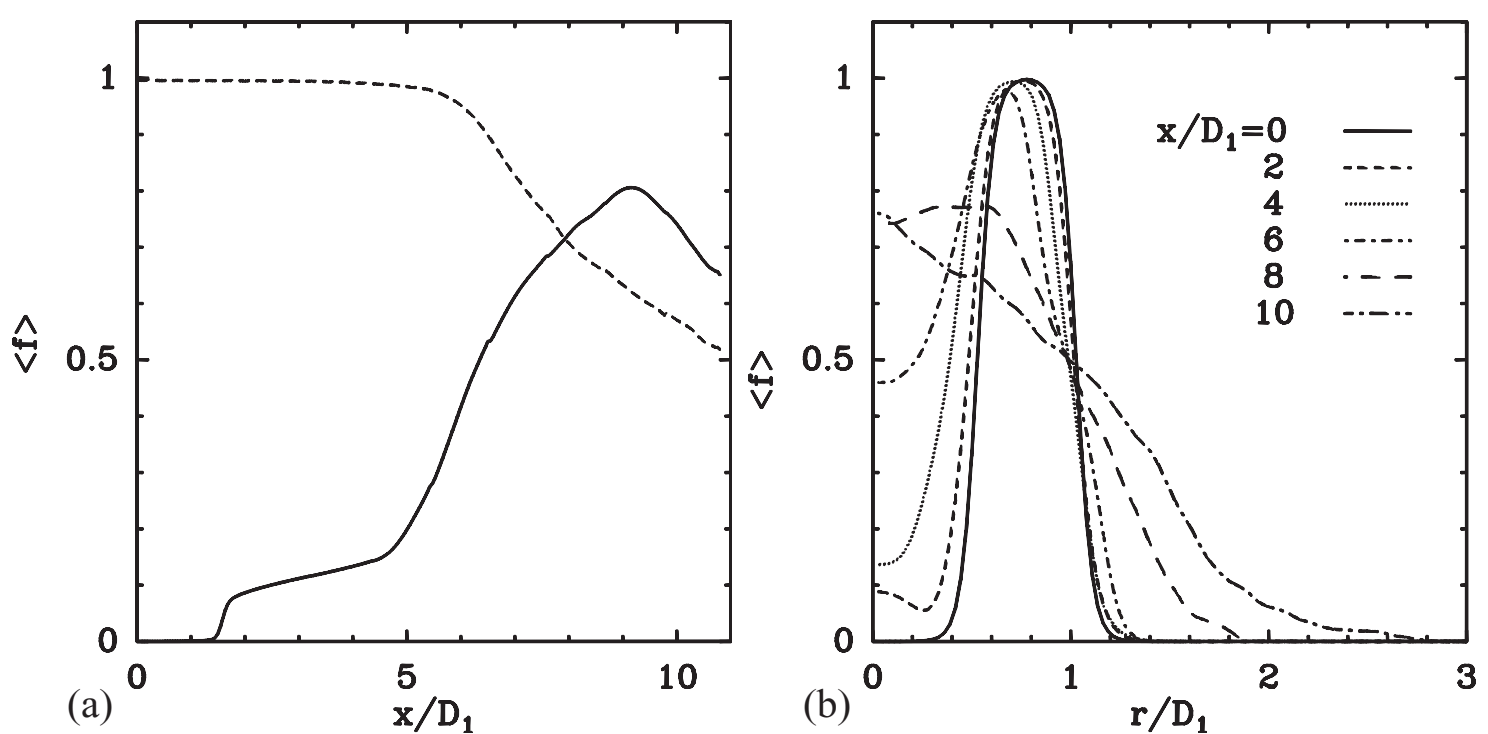

Figure 22. Mean mixture fraction. (a) Downstream evolution in both central (continuous line) and annular jet (dashed line). (b) Radial evolution at several downstream locations. 

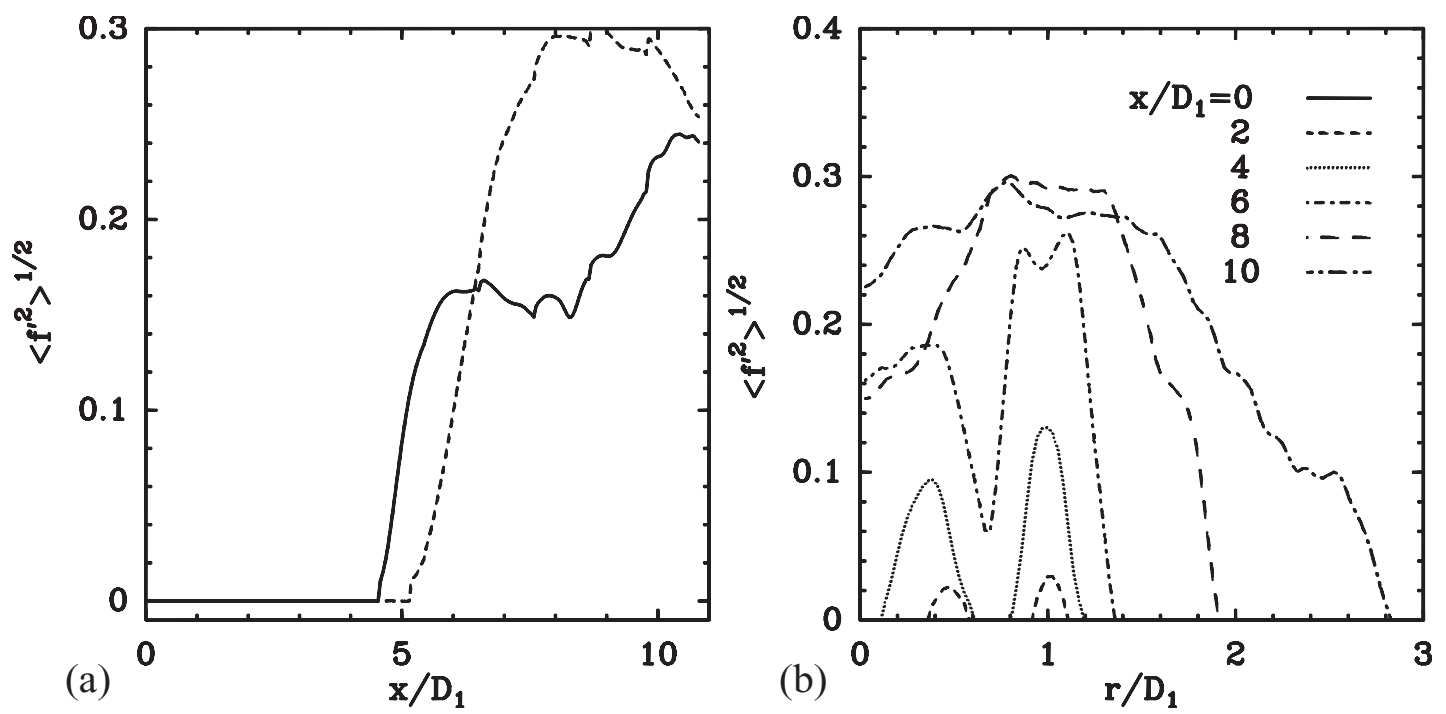

Figure 23. Rms of the mixture fraction. (a) Downstream evolution in both central (continuous line) and annular jet (dashed line). (b) Radial evolution at several downstream locations.

recirculation bubble (see figure 23(a)). Therefore, the turbulent mixing behavior in the annular jet is activated at $x / D_{1} \approx 5$, instead of $x / D_{1} \approx 6$ in the DNS1O case. This is why the mixing is considerably improved, especially in the vicinity of the end of the computational domain. Consequently, one can underline that the presence of the recirculation bubble contributes to the decrease of amounts of unmixed species B issued from the annular jet.

Figure 24(a) shows the PDFs at $x / D_{1}=2$. Species B is much earlier mixed into the center of the jet (peak of $f=0.1$ for $r / D_{1}=0$ ). However, the mixing activity is exclusively due to the molecular diffusion in the neighborhood of the recirculation bubble which is laminar for this case [2]. At $x / D_{1}=5$ (figure 24(b)), a marching mode of PDFs is obvious for $r / D_{1}>$ 0.25 . The peak of $f=0.2$ at the radial positions $r / D_{1}=0$ is located inside the bubble, which explains the persistence of a mixing activity mainly due to the molecular diffusion for $r / D_{1}<0.25$. Figure 24(c) shows PDFs at $x / D_{1}=10$. A marching behavior is recurrent at this location, with a reasonably fine homogenization of the mixing which can be much better compared with the case without recirculation bubble (DNS1O). Hence, the benefit of the recirculation bubble is considerable, from the point of view of mixture homogenization. However, at the centerline axis $r / D_{1}=0$, the probability of finding an amount of pure species $\mathrm{B}$ is again high.
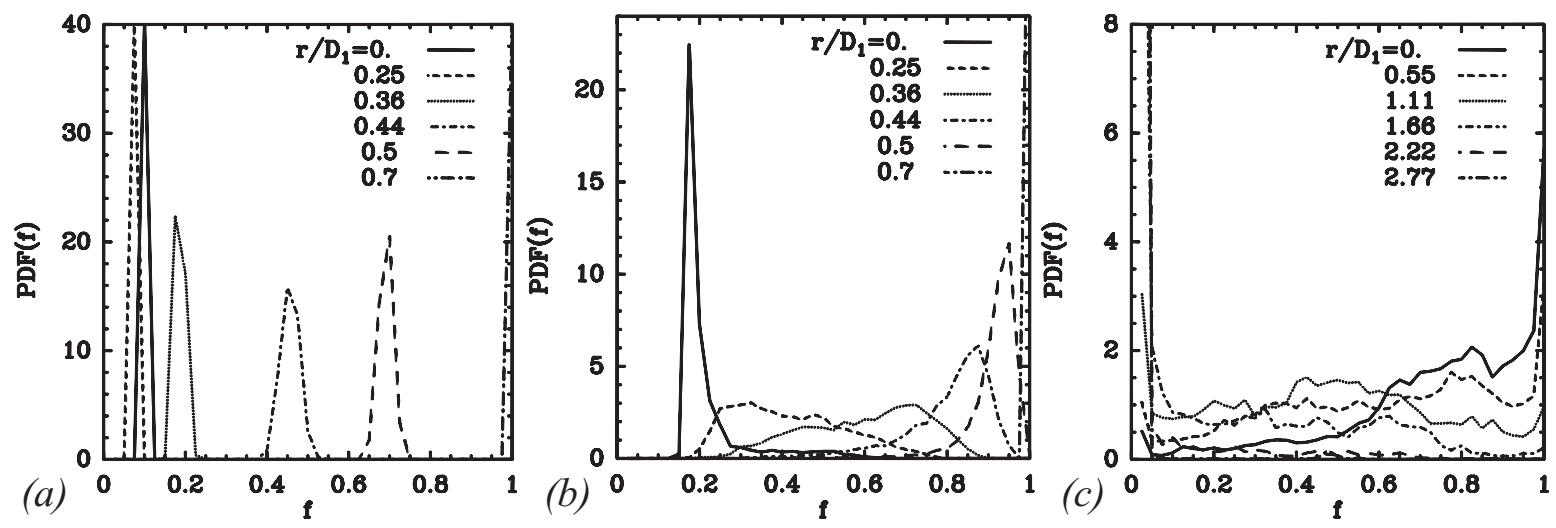

Figure 24. Variation of the mixture fraction PDFs across the mixing layer at $x / D_{1}=2$ (a), $x / D_{1}=5$ (b) and $x / D_{1}=10$ (c). 


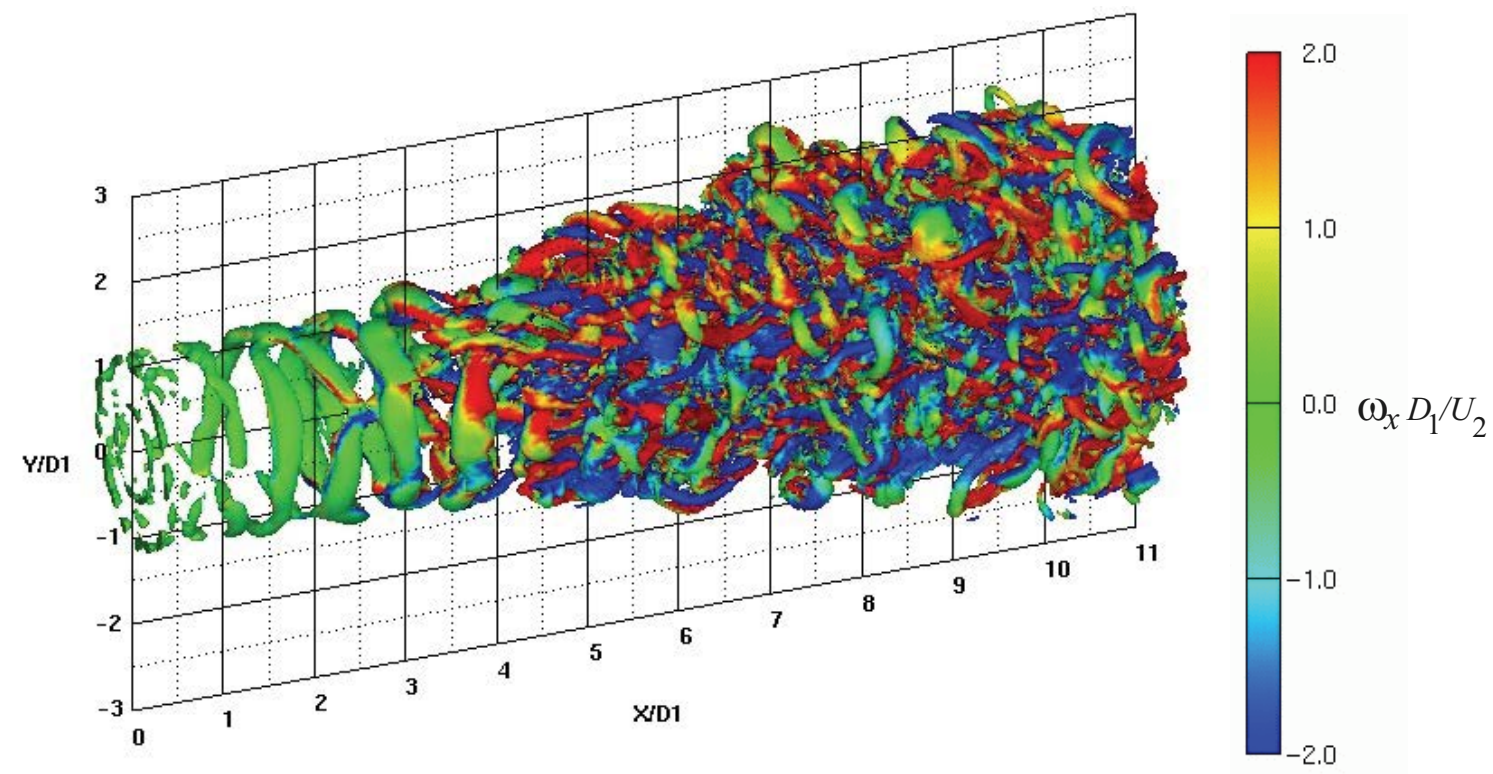

Figure 25. Positive $Q$ isosurfaces $\left(Q=0.5\left(U_{2} / D_{1}\right)^{2}\right)$ colored by the streamwise vorticity $\omega_{x}$ following the color scale shown in the figure, for DNS3O.

\subsection{Initial momentum thicknesses}

In this section, we look at the influence of the momentum thicknesses on the flow dynamics and mixing. Parameters correspond to run DNS3O. The same inlet parameters as for DNS1O are used, except for the initial values of the inner and outer momentum thicknesses: $R_{1} / \theta_{01}=$ $R_{1} / \theta_{02}=12.5$ in DNS1O, against 25 in DNS3O. Before dealing with the mixing behavior in this case, it is of interest to illustrate the structure of the turbulent flow in DNS3O.

We display isosurfaces of positive $Q$ in figure 25. It exhibits the fine structure of the shear layers, with some evidence of distinct large rollers and smaller scale vortices. The scenario of the flow transition is obviously similar to that of DNS1O. Indeed, both the KelvinHelmholtz vortices of the inner and outer jets are apparent, with the indication of the 'locking' phenomenon. The streamwise vortices emerge between two consecutive Kelvin-Helmholtz vortices, followed by an abrupt increase in the level of small scale turbulence. Although DNS1O and DNS3O involve somewhat similar features, significant differences are visible, such as the earlier development of streamwise vortices at $x / D_{1}=4$, and the breakdown to significantly small scales for $x / D_{1}>7$. Moreover, figure 26 shows the enhancement of

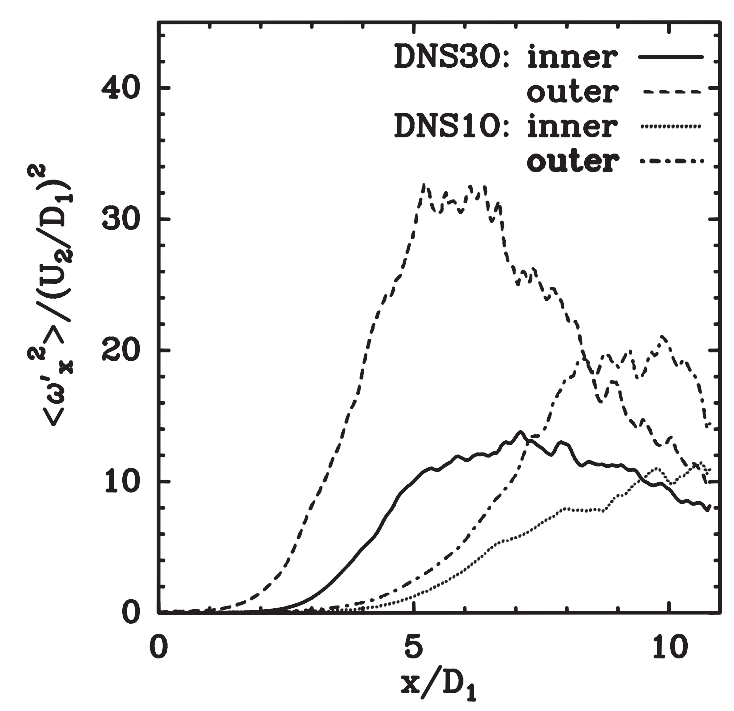

Figure 26. Downstream evolution of $\left\langle\omega_{x}^{\prime 2}\right\rangle(x, r)$ at the inner and outer shear layers for DNS1O and DNS3O. 

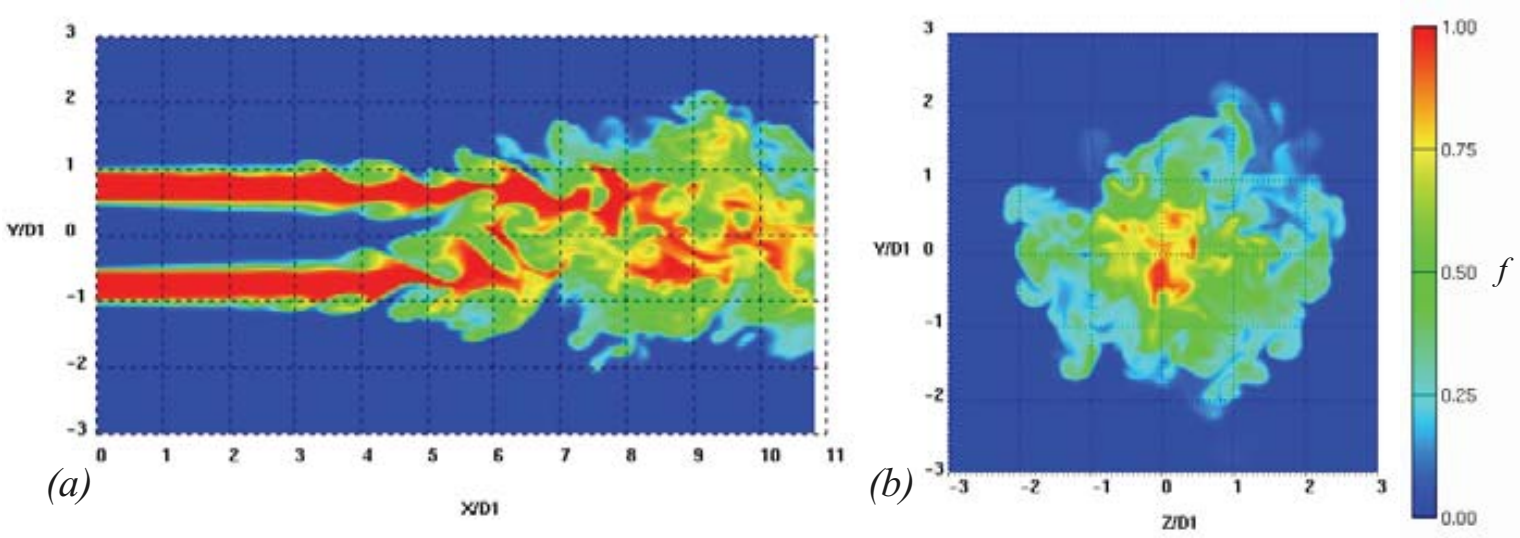

Figure 27. Instantaneous contours of mixture fraction for the DNS3O case: (a) in the central plane and (b) in the transverse section located at $x / D_{1}=10 . f$ varies from 0 (blue) to 1 (red) following the color scale shown in the figure.

$\left\langle\omega_{x}^{\prime 2}\right\rangle(x, r)$ in the inner and the outer shear layers: the longitudinal stretching is thus more intense for DNS3O than DNS1O. It is in agreement with the fact that three dimensionality is observed earlier in the jet development. It was shown in a previous work [2] that the longitudinal stretching is the dominant phenomenon leading to turbulent transition.

To illustrate basic mixing features, figures 27 and 28 show visualizations of snapshots of the mixture fraction. The first mixing stage controlled by molecular diffusion is shorter than in the DNS1O case. The earlier development of Kelvin-Helmholtz vortices activates the engulfment of large amounts of fluid species into the shear layers. Mixing layers (inner and outer) merge around $x / D_{1} \approx 6$ (instead of $x / D_{1} \approx 8$ in the DNS1O case) allowing the species $B$ invasion in the jet center earlier. At the end of the domain, the mixture fraction seems much better distributed. Figure 29 shows the mean mixture fraction in the DNS_3O case. The diffusion of the laminar layer is for $x / D_{1}<4$ in this case. Species B is mixed into the axis centerline much quicker at the downstream locations $\left(x / D_{1}>4\right)$. Additionally, a large amount of species $\mathrm{B}$ invades the co-flow up to a radial position $r / D_{1} \approx 2.6$, instead of $r / D_{1} \approx 2.2$ for the DNS1O case. This spectacular spreading of the mixture is mainly due to the streamwise vortices which enhance entrainment and mixing. The discrepancy in mixturefraction rms between DNS3O (figure 30) and DNS1O, is essentially due to the transitional

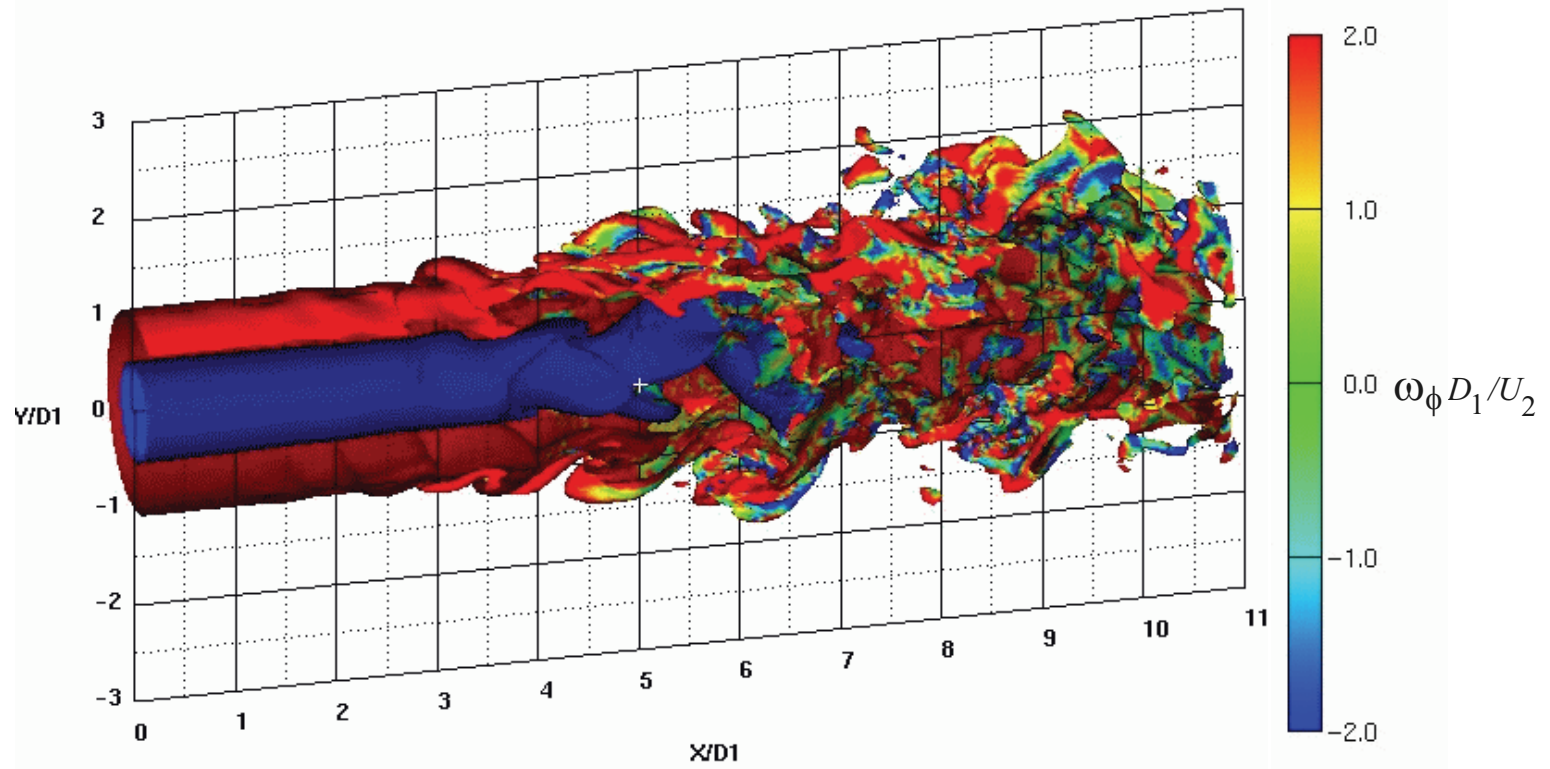

Figure 28. Cut view of $f=0.5$ mixture-fraction isosurface colored by the tangential vorticity $\omega_{\phi}$ following the color scale shown on the figure, for DNS3O. 

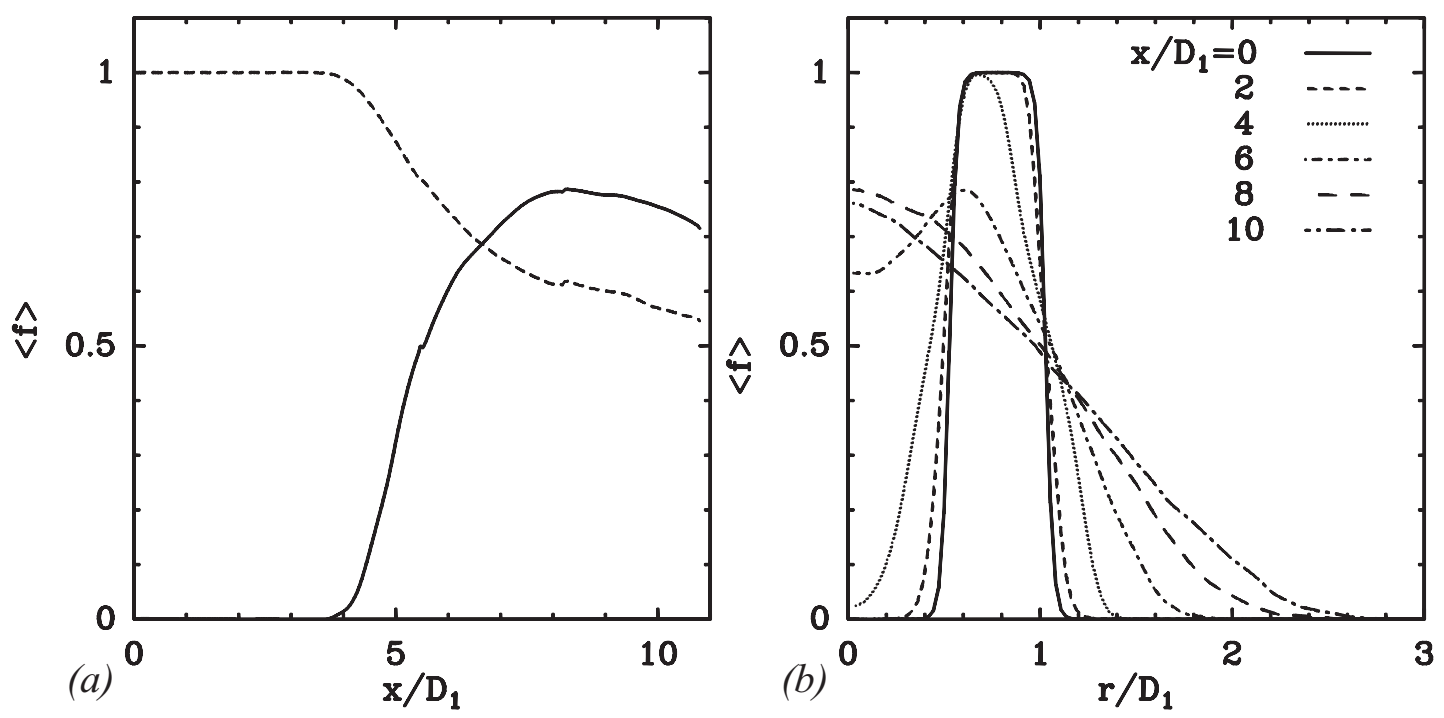

Figure 29. Mean mixture fraction. (a) Downstream evolution in both central (continuous line) and annular jets (dashed line). (b) Radial evolution at several downstream locations.

region, where large coherent vortices are different. Figure 31(a) shows PDFs of the inner mixing layer at $x / D_{1}=2$. A mixing behavior similar to that observed in the DNS1O case is obvious. However, discrepancies can be observed in the outer mixing layer (figure 31(b)). The early development of the large-scale vortices, due to the small values of $\theta_{01}$ and $\theta_{02}$, allows mixing more quickly. At $x / D_{1}=5$, the PDFs (figures 32(a) and 32(b)) are relatively nonmarching showing an important mixing activity. Moreover, figure 32(a) shows the presence of pure species A near the jet centerline for $r / D_{1}=0$ and $r / D_{1}=0.14$. Nevertheless, a marching-type PDF is obtained at $x / D_{1}=10$ showing homogeneous mixing. The probability of finding pure species $\mathrm{B}(f=1)$ is much smaller at this downstream location, in contrast the DNS1O case.

\subsection{Ratio of outer to inner diameters}

In this section the ratio of the outer to the inner diameters is changed, in order to analyze its effect on the flow and the mixing process. The inner diameter is increased until a value of $1.3 D_{1}$ while the outer diameter is fixed to its initial value $2 D_{1}$. In this case, the inner and the
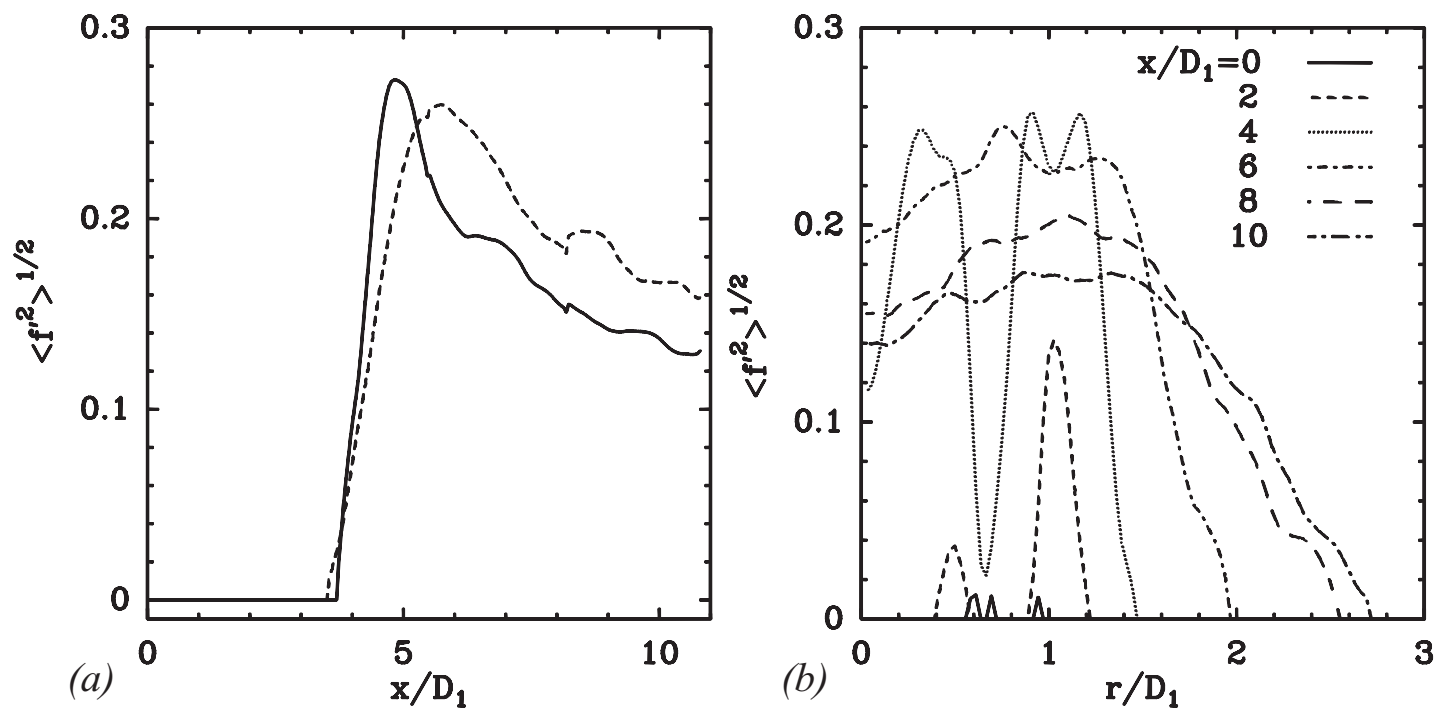

Figure 30. Rms of the mixture fraction. (a) Downstream evolution in both central (continuous line) and annular jets (dashed line). (b) Radial evolution at several downstream locations. 

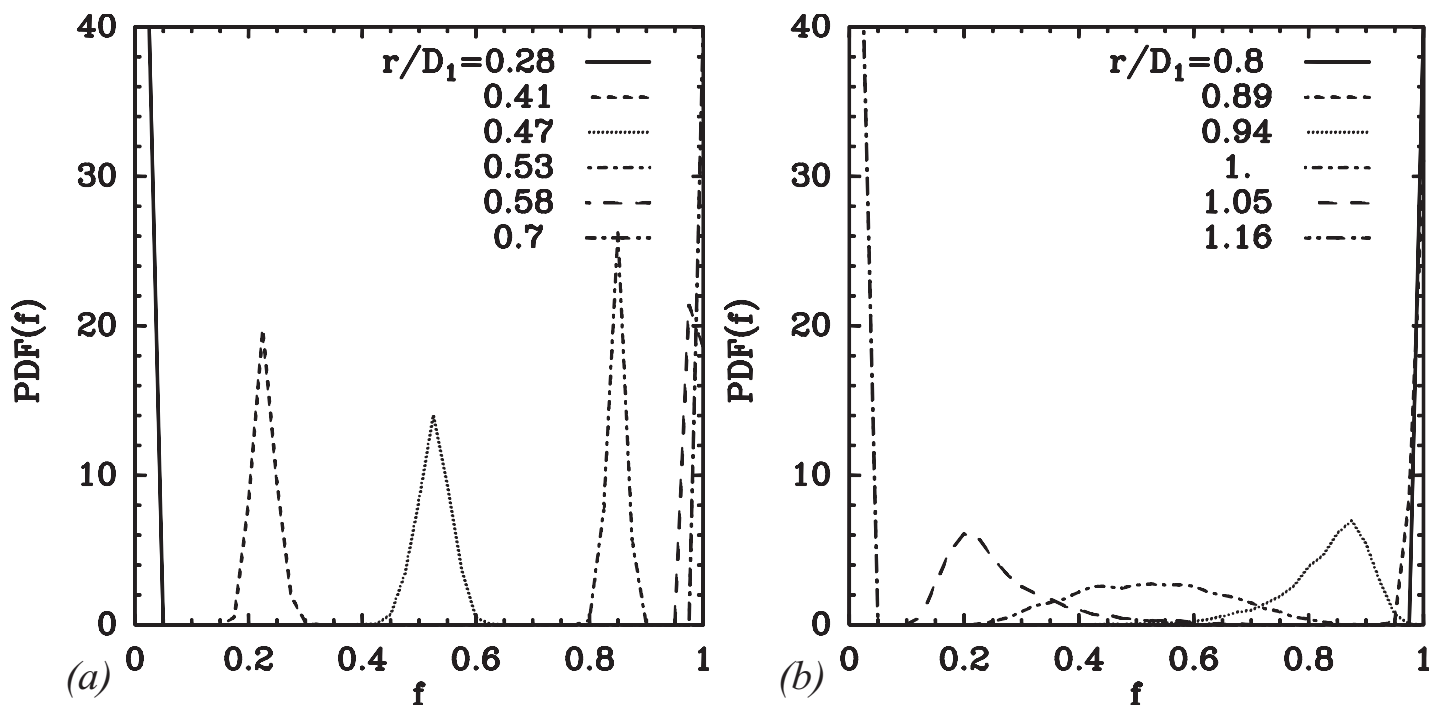

Figure 31. The variation of the mixture fraction PDFs across the inner mixing layer (a) and the outer mixing layer (b) at $x / D_{1}=2$.

outer momentum thicknesses are the same as those of the DNS3O. Figure 34 shows the first picture of the flow dynamics for DNS4O. We recover the same scenario as for DNS3O, which leads to a jet breakdown into fully developed turbulence: the laminar diffusion layer, followed by the formation of Kelvin-Helmholtz and streamwise vortices and catastrophic breakdown into small scales.

There are however differences in the statistics between DNS3O and DNS4O. Figure 35 shows $\left\langle\omega_{x}^{\prime 2}\right\rangle$ in the inner and outer shear layers for the two cases. The evolution is the same in the outer shear layer, but in the inner layer the growth is quicker in the DNS4O case. This is due to the proximity between the two layers in this configuration. Thus, instabilities are easily transmitted from the outer to the inner layer, which allows an earlier transition. Figure 36 gives a first picture of the mixing scenario, which can be explained as above: molecular diffusion at the onset of the jet development and domination of the turbulent mixing far downstream. Moreover, unmixed species B is obviously much less present for $x / D_{1}>6$, in contrast to the DNS3O case (figure 27). This is certainly due to the intensification of streamwise vortices in the inner jet. Figure 37(b) shows the mean mixture fraction profiles at several downstream positions. Like DNS3O, the diffusion of the laminar layer is limited to $x / D_{1}<4$. Species
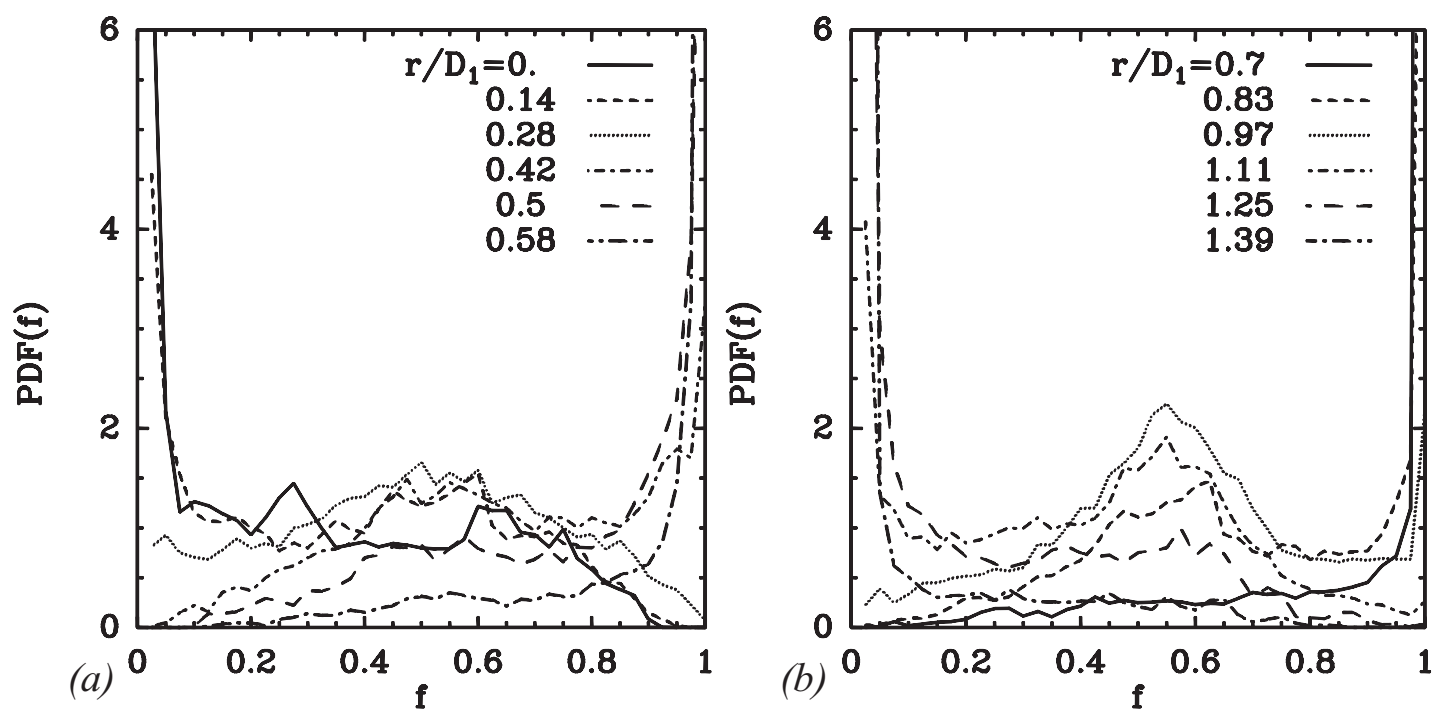

Figure 32. Variation of the mixture fraction PDFs across the inner mixing layer (a) and the outer mixing layer (b) at $x / D_{1}=5$. 


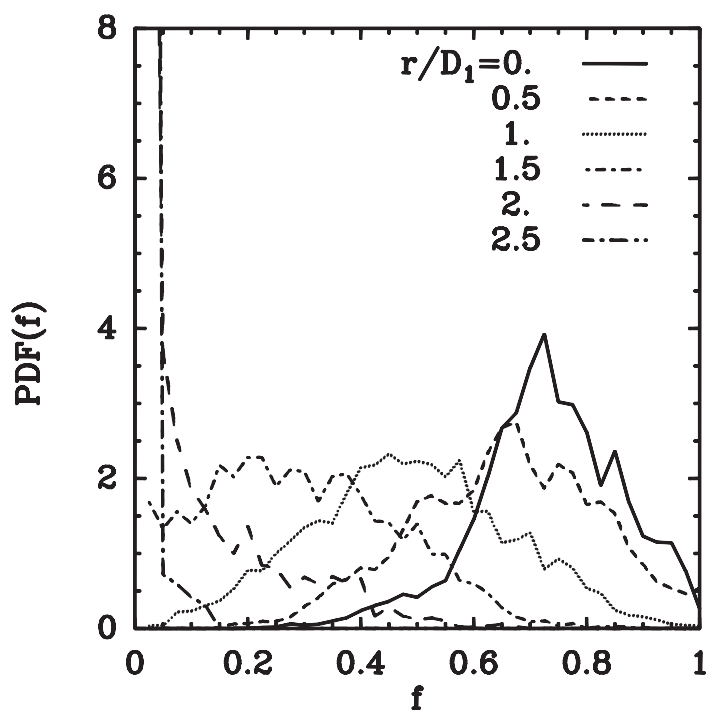

Figure 33. The variation of the mixture fraction PDFs across the mixing layer at $x / D_{1}=10$.

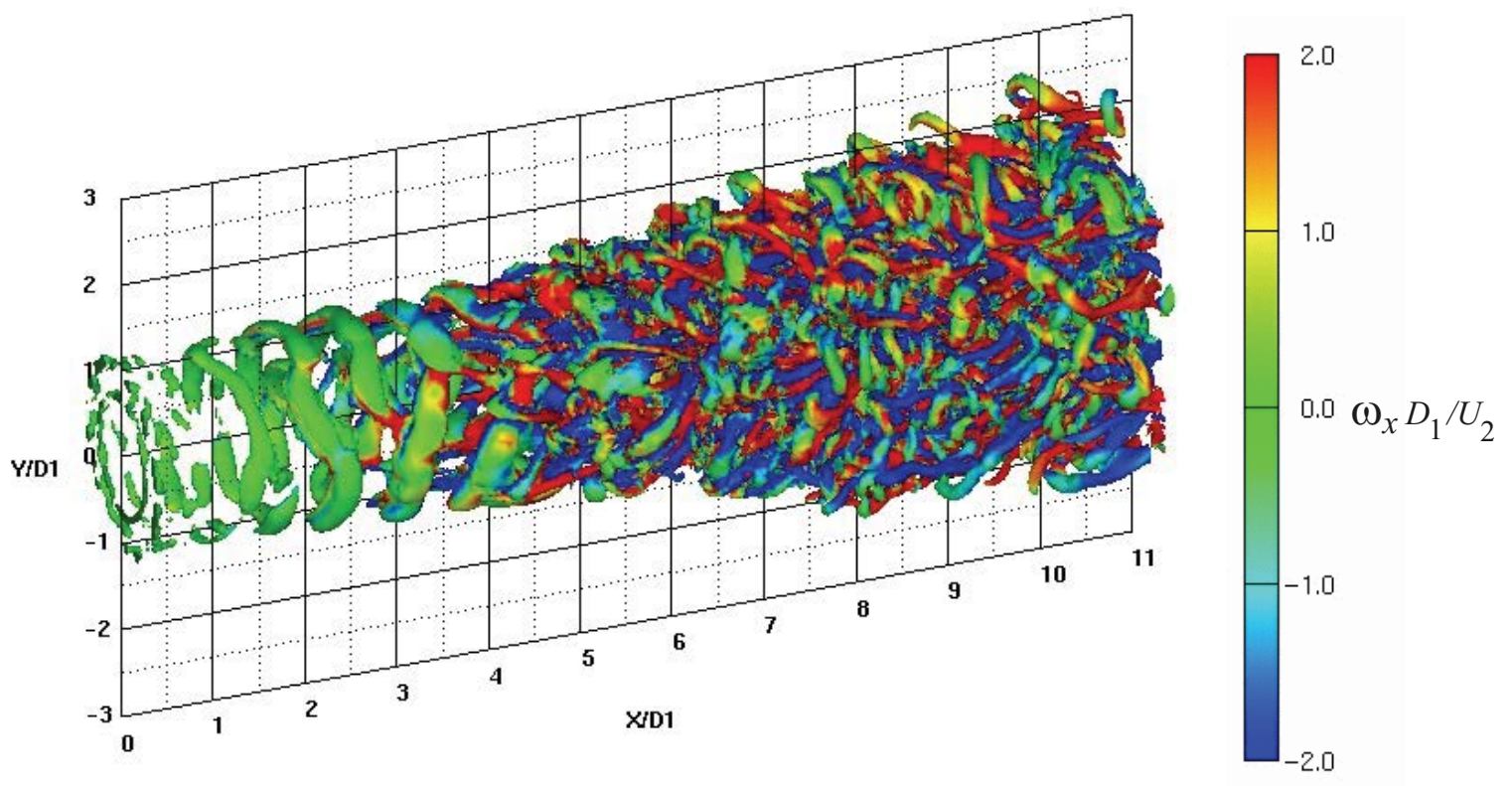

Figure 34. Positive $Q$ isosurfaces $\left(Q=0.5\left(U_{2} / D_{1}\right)^{2}\right)$ colored by the streamwise vorticity $\omega_{x}$ following the color scale shown in the figure, for DNS4O.

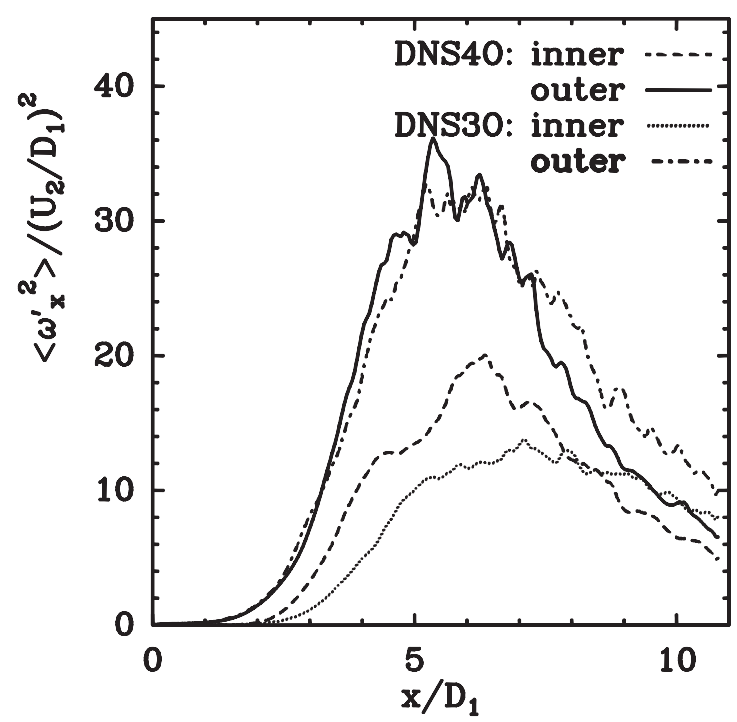

Figure 35. Downstream evolution of $\left\langle\omega_{x}^{\prime 2}\right\rangle(x, r)$ at the inner and outer shear layers for DNS4O and DNS3O. 

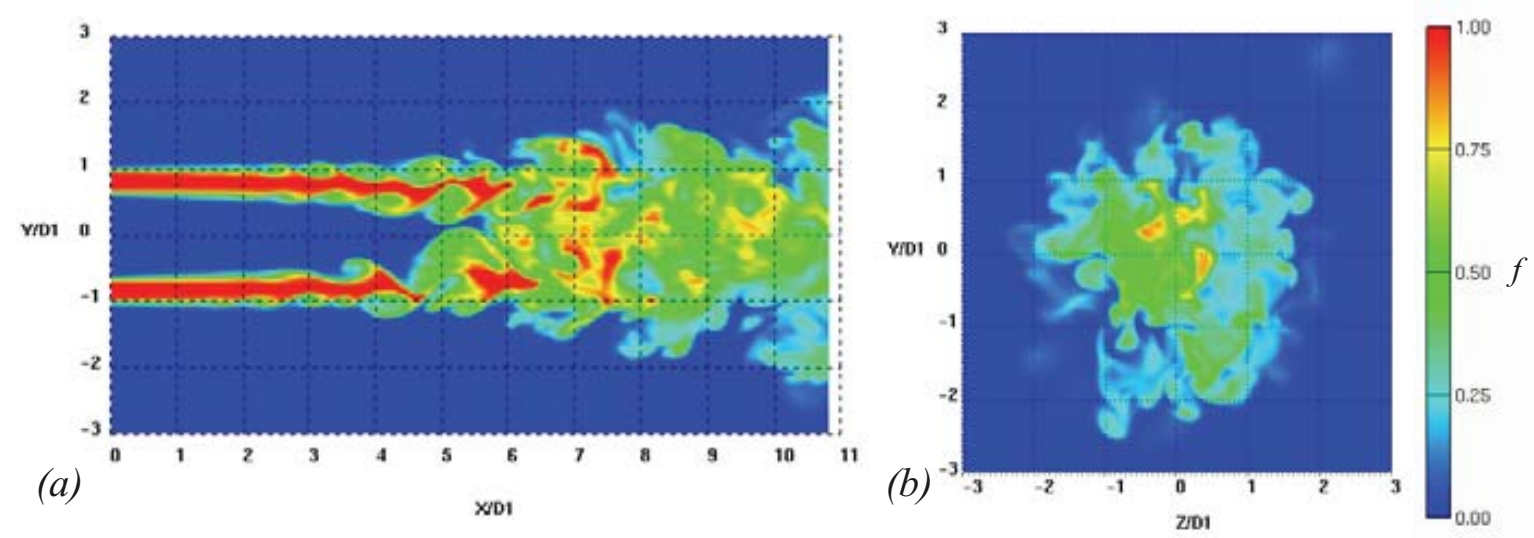

Figure 36. Instantaneous contours of mixture fraction for the DNS4O case: (a) in the central plane and (b) in the transverse section located at $x / D_{1}=10 . f$ varies from 0 (blue) to 1 (red) following the color scale shown on the figure.

B is mixed into the axis centerline quicker than in the DNS30 case (figure 37(a)). This is certainly due to the increasing intensity of streamwise vortices in the inner jet. Figure 38 shows the rms mixture fraction. The turbulent mixing activity in the outer jet starts quicker than in the DNS3O case. Nevertheless, it begins later in the central jet than in the DNS3O case, as the inner mixing layer is more distant. We believe also that the difference in rms mixture fraction statistics is due to the difference in their transitional states. At $x / D_{1}=5$, a non-marching-type PDF is obvious (figure 39(a)). It indicates a mixing activity that is more intense than that of the DNS3O case at this downstream location. Here, PDFs are calculated only in the inner mixing layer, since the outer mixing layer development is not very much influenced by this reduction in the diameter ratio. At $x / D_{1}=10$, the same behavior (marching type) of the PDFs as in DNS3O is obtained (figure 39(b)). We can also note that species B are completely mixed at this downstream location, since the probability of finding $f>0.9$ is zero.

\subsection{Global comparison between the different cases}

In this section we examine the global mixedness quantitatively for all the cases. Global mixedness can be considered as a measure of the mixing, easy to compute with respect to the mixing efficiency which is determined from the behavior of interfaces stirred by the fluid. This quantity
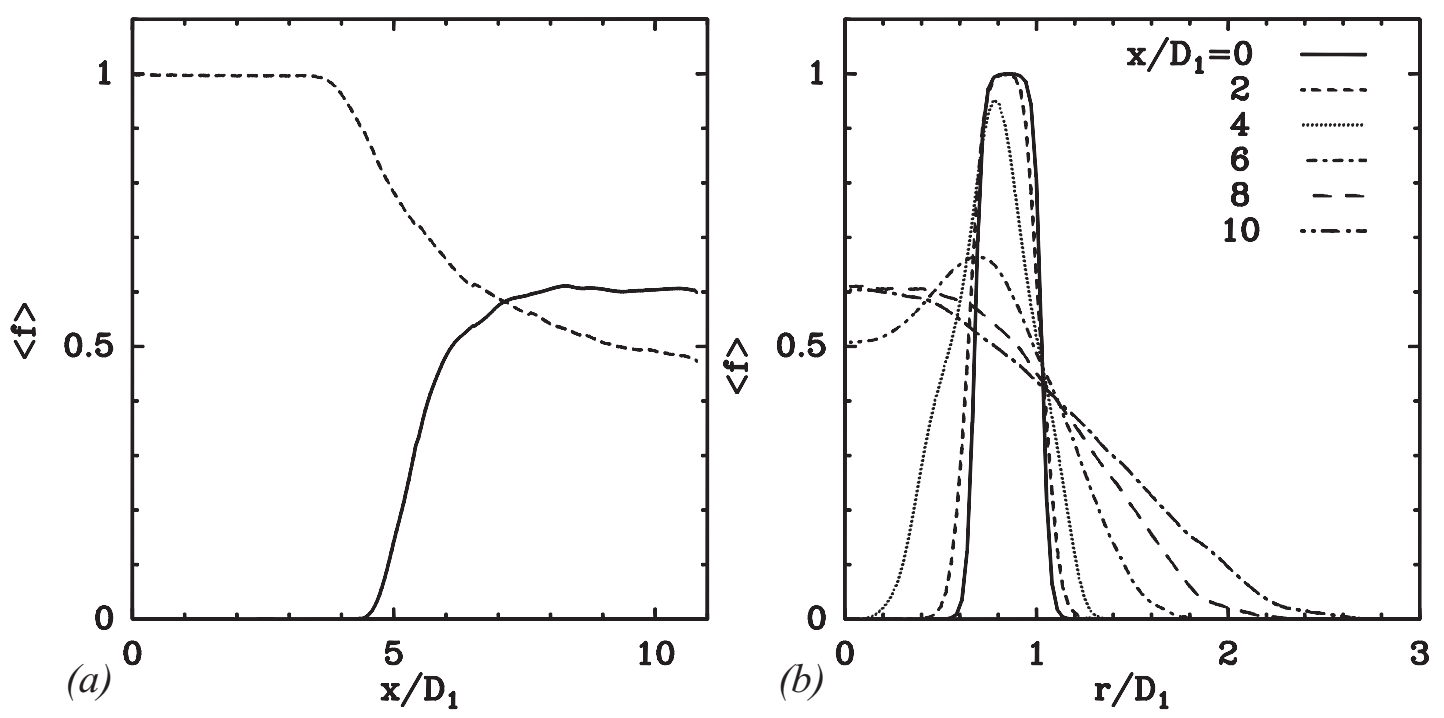

Figure 37. Mean mixture fraction. (a) Downstream evolution in both central (continuous line) and annular jets (dashed line). (b) Radial evolution at several downstream locations. 

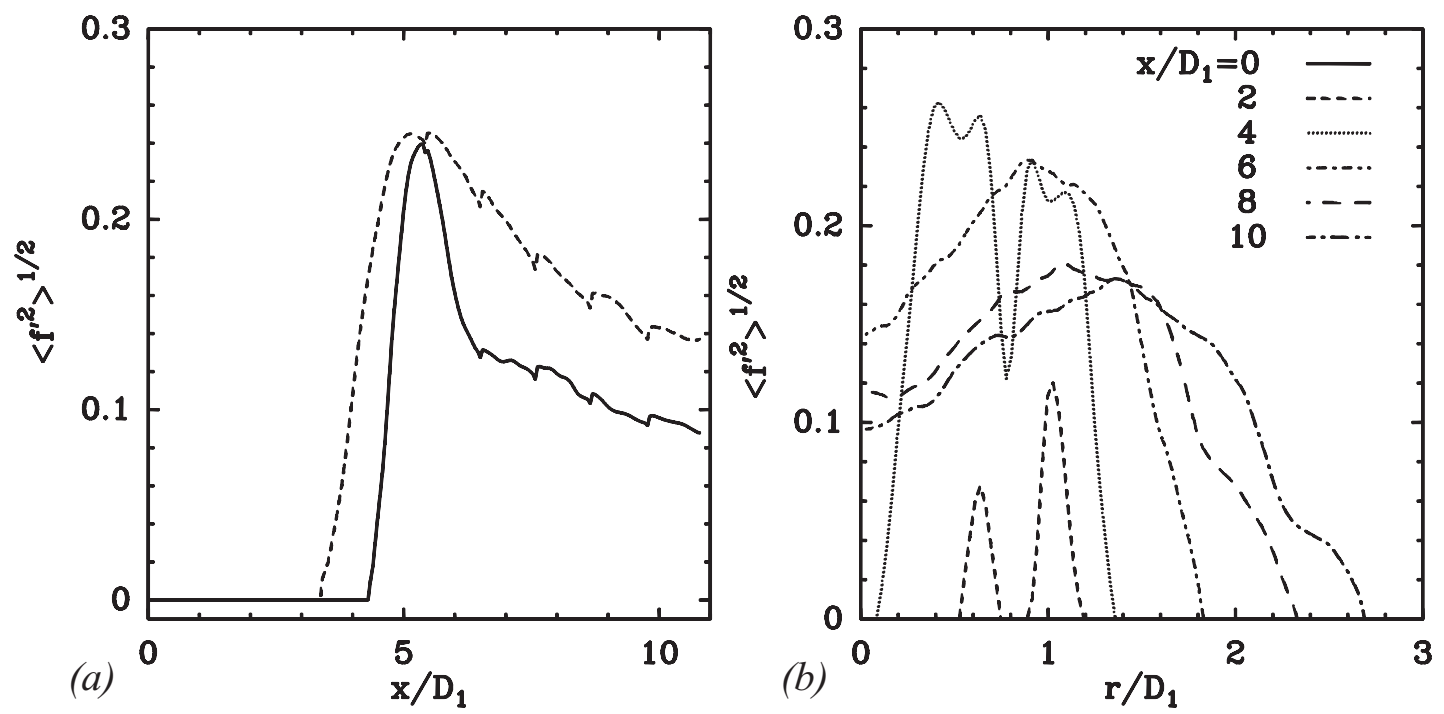

Figure 38. Rms of the mixture fraction. (a) Downstream evolution in both central (continuous line) and annular jet (dashed line). (b) Radial evolution at several downstream locations.

$Z(x)$, introduced by Cetegen and Mohamad [29], is defined as the second moment of the mixture fraction:

$$
Z(x)=\frac{4}{S} \int_{S}\langle f(x, r)\rangle(1-\langle f(x, r)\rangle) r \mathrm{~d} r \mathrm{~d} \phi .
$$

In equation (5), $S$ is the radial section where the integration is performed and $\phi$ is the angle in cylindrical coordinates $(x, r, \phi)$. In the integration the radius of $S$ is equal to $3 D_{1}$ to include all the region where $\langle f\rangle \neq 0$ at the exterior of the computational domain in all the studied cases. $Z(x)$ ranges from 0 for completely unmixed fluids (only $f=0$ or $f=1$ in the section) to 1 for complete mixing. Note that this definition is appropriate when the complete mixing is for a bi-molecular mixing. For example, it is a good indicator if each species is needed with the same proportion by the chemical reaction to be considered. Figure 40 shows the evolution of $Z(x)$ for the different cases examined in the present study. It is clear that the cases DNS2O, DNS3O and DNS4O lead to a finer mixing. The benefit in mixing for DNS3O and DNS4O is due to their early transition states and hence the early development of the turbulent mixing activity. In DNS3O, the fast transition state is due to the values of the momentum thicknesses, which allow the appearance of the streamwise vortices earlier. For the case with the recirculation bubble
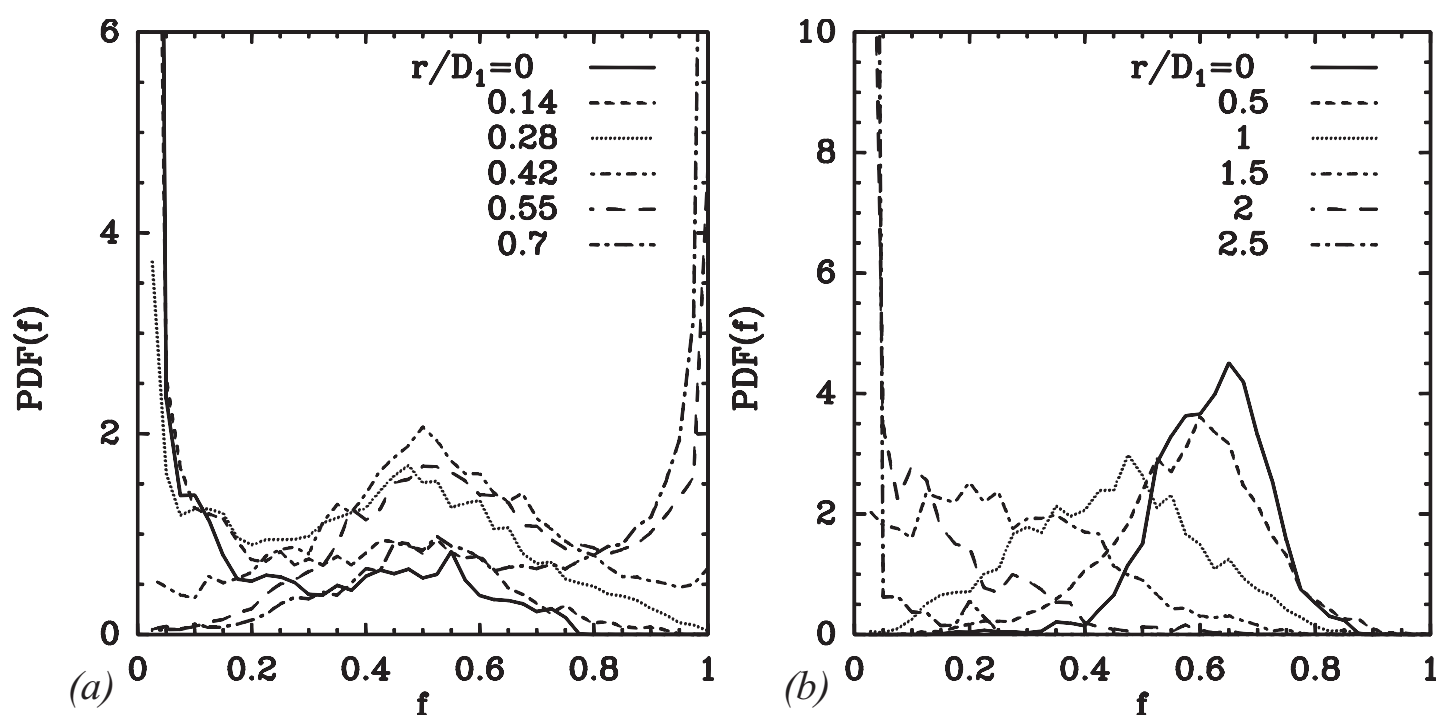

Figure 39. Variation of the mixture fraction PDFs across the mixing layer at $x / D_{1}=5$ (a) and $x / D_{1}=10$ (b). 


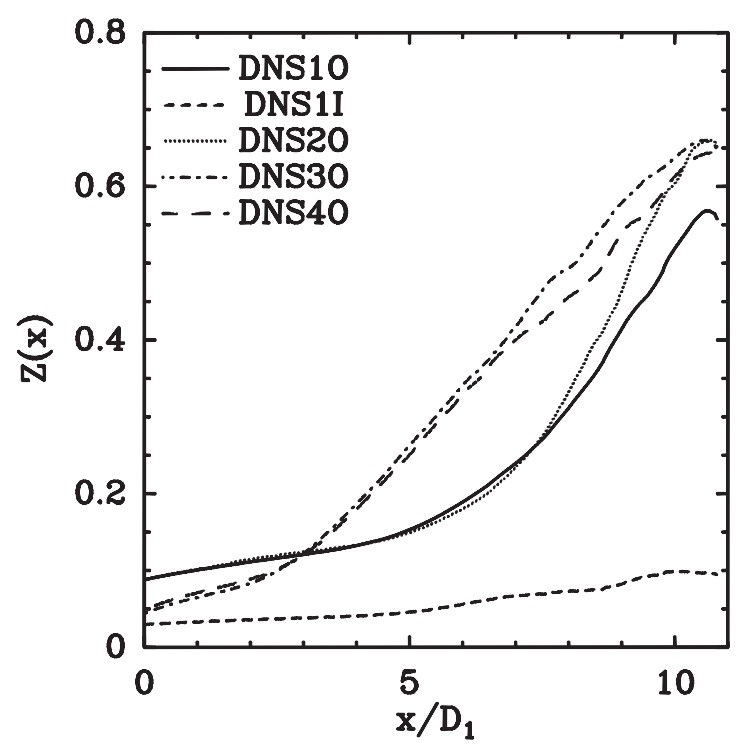

Figure 40. Mixedness of the different cases.

(DNS2O), the evolution of $Z(x)$ is the same as for DNS1O for $x / D_{1}<6$. For $x / D_{1}>6, Z(x)$ increases more rapidly because the bubble presence enhances the intensity of the streamwise vortices. Run DNS1I is very unfavorable, since the seeding of the inner jet by species B prevents the outer shear layer to participate in the mixing behavior.

\section{Conclusions}

In the present study, the mixing behavior in free round coaxial jets has been investigated by means of direct numerical simulations (DNS). Five computations have been performed to assess the effect of upstream conditions upon the turbulent vortices and structures, and hence on the passive scalar mixing processes. The mixing between two species A and B seeded in the coaxial jets is tracked through the spatial and temporal evolution of the mixture fraction field. The spatially developing approach has permitted to take into account the stages of the mixing process until adequate stirring conditions are obtained. Of particular importance is the appearance of a fully developed turbulent state, as far as dynamic quantities are concerned. The results presented here highlight the fact that the turbulent-mixing process in coaxial jets is affected by the upstream conditions, which modify the transitional state. The peak values of the mixture fraction rms $\left\langle f^{\prime 2}\right\rangle^{1 / 2}$ are not the same in the far field for runs DNS1O and DNS3O. Furthermore, the PDF distributions of $f$ at far downstream locations indicate notable differences. We think these differences are due to different vortical behaviors, especially the intense production of the streamwise vortices, as obtained in runs DNS2O and DNS4O. We have also shown that, although, a self-similar state is achieved, the mean gradients of the upstream velocity profile affects the mixing behavior even at far locations. Comparison between DNS1O and DNS1I has highlighted that the fluid issued from the central jet remains confined, whereas the annular fluid invades the center jet in a straightforward manner. A great contrast between the width of the jet and the spreading of the mixture has been observed in the case of DNS1I, since only the inner shear layer contributes to the mixing process. Computing the global mixedness (which can be regarded as mixing efficiency) has shown the disadvantage of this configuration. Hence it may be recommended for combustion application to avoid seeding in the coaxial jet, as in DNS1I. Spots of pure species at the exit of the computational domain, shown to be due to the annular jet, are observed in runs DNS1O, DNS2O, DNS3O. Modifications of the inner jet diameter can lead to the decrease of amounts 
of pure species in the annular fluid far downstream, as it is shown in run DNS4O. Of course, increasing the central jet diameter allows us to bring closer the shear layers, which induces a much quicker appearance of the inner streamwise vortices and thus improves the turbulent mixing activity, as it is quantitatively shown by the global mixedness.

\section{Acknowledgements}

We thank one referee for a very thorough correction of the paper.

Part of the computations were carried out at the Institut du Développement et des Ressources en Informatique Scientifique (IDRIS, France).

\section{References}

[1] da Silva, C.B., Balarac, G. and Métais, O. 2003, Transition in high velocity ratio coaxial jets analysed from direct numerical simulations. Journal of Turbulence, 4(24), 1-18.

[2] Balarac, G. and Métais, O., 2005, The near field of coaxial jets: a numerical study. Physics of Fluids, 17(065102), $1-14$.

[3] Salvetti, M.V., 1999, Effects of the velocity ratio on vorticity dynamics and mixing in coaxial jet flows. Proceedings 1st International Conference on Turbulence and Shear Flows Phenomena, Santa Barbara, CA, USA.

[4] Jahnke, S., Kornev, N., Tkatchenko, I., Hassel, E. and Leder, A., 2005, Numerical study of influence of different parameter on mixing in coaxial jet mixer using LES. Heat Mass Transfer, 41, 471-481.

[5] Villermaux, E. and Rehab, H., 2000, Mixing in coaxial jets. Journal of Fluid Mechanics, 425, 161-185.

[6] Ferrao, P.C., Heotor, M.V., Matos, M.F. and Salles, R.K., 1999, Turbulent scalar mixing in coaxial jet flows, Proceedings 1st International Conference on Turbulence and Shear Flows Phenomena, Santa Barbara, CA, USA.

[7] Ritchie, B.D., Mujumdar, D.R. and Seitzman, J.M., 2000, Mixing in coaxial jets using synthetic jet actuators. AIAA Paper.

[8] Kurimoto, N., Suzuki, Y. and Horiuchi, N., 2001, Active control of coaxial jet mixing and combustion with arrayed micro actuators, Experimental Heat Transfer, Fluid Mechanics, and Thermodynamics.

[9] Suzuki, Y., Kasagi, N., Horiuchi, Y. and Nagoya, D., 2000, Synthesized mixing process in an actively-controlled confined coaxial jet. 4th JSME-KSME Thermal Engineering Conference.

[10] Lesieur, M., Métais, O. and Comte, P., 2005, Large-Eddy Simulations of Turbulence. Cambridge, UK: Cambridge University Press.

[11] Koochesfahani, M.M. and Dimotakis, P.E., 1986, Mixing and chemical reactions in a turbulent liquid mixing layer. Journal of Fluid Mechanics, 170, 83-112.

[12] Orlanski, I., 1976, A simple boundary condition for unbounded hyperbolic flows. Journal of Computational Physics, 21, 251-269.

[13] Gonze, M.A., 1993, Simulation numérique des sillages en transition à la turbulence, PhD Thesis, Institut National Polytechnique de Grenoble.

[14] da Silva, C.B. and Métais, O., 2002, Vortex control of bifurcating jets: a numerical study. Physics of Fluids, 14(11), 3798-3819.

[15] Hirsch, C., 2002, Numerical Computation of Internal and External Flows, vol. 2. New York: Wiley.

[16] da Silva, C.B. and Métais, O., 2002, On the influence of coherent structures upon interscale interactions in turbulent plane jets. Journal of Fluid Mechanics, 473, 103-145.

[17] Balarac, G. and Si-Ameur, M., 2005, Mixing and coherent vortices in turbulent coaxial jets. Comptes Rendus Mécanique, 333, 622-627.

[18] Hunt, J.C.R., Wray, A.A. and Moin, P., 1988, Eddies, stream, and convergence zones in turbulent flows. Annual Research Briefs, Center for Turbulence Research, Stanford.

[19] Dubief, Y. and Delcayre, F., 2000, On coherent vortex identification in turbulence. Journal of Turbulence, 1(11), 1-22.

[20] Balarac, G. and Métais, O., 2004, Coherent vortices in coaxial jets. In H. I. Anderson and P.-A. Krogstad (Eds.), Advances in Turbulence X. Barcelona: CIMNE

[21] Liepmann, D. and Gharib, M., 1992, The role of the streamwise vorticity in the near-field entrainment of a round jet. Journal of Fluid Mechanics, 245, 643-668.

[22] Rehab, H., 1997, Structure de l'écoulement et mélange dans le champ proche des jets coaxiaux. PhD Thesis, Institut National Polytechnique de Grenoble.

[23] Rehab, H., Villermaux, E. and Hopfinger, E.J., 1997, Flow regimes of large-velocity-ratio coaxial jets. Journal of Fluid Mechanics, 345, 357-381. 
[24] Angele, K.P., Kurimoto, N., Suzuk, Y. and Kasagi, N., 2005, Three-dimensional vortical structures in a coaxial jet manipulated with micro-actuators. Proceedings of 6th World Conference on Experimental Heat Transfer, Fluid Mechanics, and Thermodynamics, Matsushima, Miyagi, Japan.

[25] Stanley, S.A., Sarkar, S. and Mellado, J.P., 2002, A study of the flow-field evolution and the mixing in a plane turbulent jet using direct numerical simulation. Journal of Fluid Mechanics, 450, 377-407.

[26] Buch, K.A. and Dahm, W.J.A., 1998, Experimental study of the fine scale structure of conserved scalar mixing in turbulent shear flows: part II. Sc $\approx 1$. Journal of Fluid Mechanics, 364, 1-29.

[27] Métais, O. and Lesieur, M., 1992, Spectral large-eddy simulations of isotropic and stably-stratified turbulence. Journal of Fluid Mechanics, 239, 157-194.

[28] Lesieur, M. and Rogallo, R., 1989, Large-eddy simulation of passive scalar diffusion in isotropic turbulence. Physics Fluids A, 1(9), 718-722.

[29] Cetegen, B.M. and Mohamad, N., 1993, Experiments on liquid mixing and reaction in a vortex. Journal of Fluid Mechanics, 249, 391-414. 